\title{
Validation of the uncertainty bounds on modal parameters identified with the SSI-COV method
}

\author{
Yeny V. Ardila A. ${ }^{a}\left(\mathbb{D}\right.$, Iván D. Gómez Araújo ${ }^{a *}\left(\mathbb{D}\right.$, Jesús D. Villalba-Morales ${ }^{b}(\mathbb{D}$, Luis A. Aracayoc \\ a Engenharia Civil e Infraestrutura, Universidade Federal da Integração Latino-Americana (UNILA), Foz do Iguaçu, Brasil. Email: \\ yva.ardila.2019@aluno.unila.edu.br, ivan.araujo@unila.edu.br \\ ${ }^{b}$ Departamento de Ingenieria Civil, Pontificia Universidad Javeriana (PUJ), Bogotá, Colombia. Email: jesus.villalba@javeriana.edu.co \\ ${ }^{c}$ Centro de Competência em Estruturas de Barragens (EB.DT-FPTI), Foz do Iguaçu, Brasil. Email: luis.aracayo@pti.org.br \\ * Corresponding author
}

https://doi.org/10.1590/1679-78256725

\begin{abstract}
Applying the Covariance-driven Stochastic Subspace Identification method (SSI-COV) involves uncertainties in the numerical process of identifying modal parameters in a system. The main issue is that the method does not compute the uncertainty in the results, which is required in some problems such as outlier detection. Currently, one method is available to assess the uncertainty in modal parameters obtained using the SSI-COV. This method based on the sensitivity analysis of the modal parameters to perturbations in the collected data and is efficient but highly complex for its computational implementation. Thus, this article presents a validation of the uncertainty results obtained with this procedure through the uncertainty limits obtained using the Bootstrap technique. The validation is performed on the modal parameters of a numerical beamtype structure with controlled noise levels and the modal parameters of a concrete block of the Itaipu Hydroelectric Dam. The uncertainty limits obtained using the two methodologies showed similarities in the two examples, which allowed validating the sensitivity analysis procedure.
\end{abstract}

\section{Keywords}

Uncertainty, bootstrap, sensitivity, modal parameter, identification

\section{Graphical Abstract}

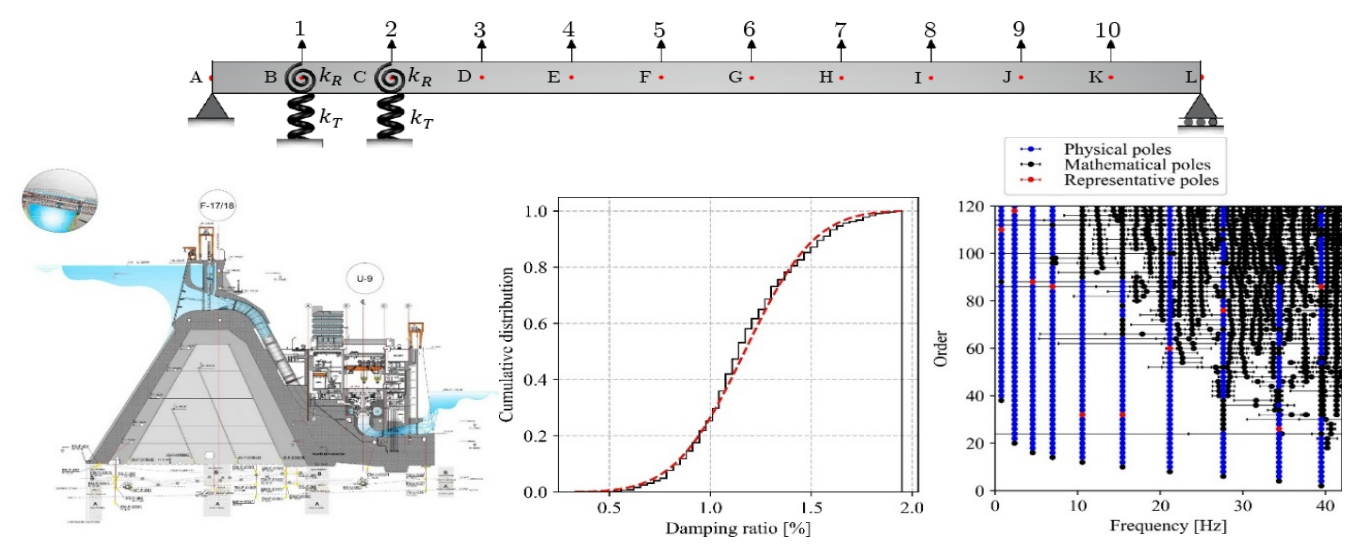




\section{INTRODUCTION}

Due to technological advances in measurement devices and the development of methods for automatized operational modal analysis (OMA), it is nowadays possible to extract the dynamic characteristics of large civil structures (such as buildings, bridges, and dams) under normal operating conditions from the response measurements caused by ambient vibrations. One of the main issues is selecting a reliable method to identify the structure's modal parameters (natural frequencies, damping ratios, and mode shapes). In that sense, results from applying the covariance-driven stochastic subspace identification method (SSI-COV) for built structures (Magalhães et al., 2008; Brownjohn et al., 2010; Liu et al., 2012; Pereira et al., 2018) show that the technique provides accurate results efficiently and robustly. However, the presence of several sources of error (e.g., finite number of data, stochastic nature of the ambient loading, nonlinearities, instrumentation noise) induce a certain degree of uncertainty in the identified modal parameters, and the SSI-COV algorithm does not provide the information for evaluating the accuracy in the results.

Different types of uncertainties impact the SSI-COV method's stages during its application. At the beginning of the process, the uncertainty associated with the collected data affects the estimation of the covariance functions; then, the accumulated uncertainty propagates through each of the stages of system identification and finally to the modal parameters. Thus, the last stage of the method inherits the uncertainties from the previous step. Reynders et al. (2008) derived a numerical procedure to estimate the uncertainties of the modal parameters obtained at some given model order from the SSI-COV method to deal with that aspect. The approach quantifies the degree of uncertainty in the modal parameters by computing their covariances, estimated through a sensitivity analysis. The procedure was applied in the operational modal analysis of a steel transmitter mast to show its practicability. However, the direct implementation of this methodology is exceedingly expensive, mainly when the dynamic test implies an extensive sensor network and the use of high model order.

The quantification of modal parameter uncertainties plays a significant role for different purposes. For instance, in (Pereira et al., 2020), modal parameter uncertainties identified in continuous dynamic monitoring were used to remove outliers during modal tracking. Döhler et al. (2015) improved the results of a damage localization method by including the computed uncertainty levels in modal parameters. Verboven et al. (2004) performed an EMA test, finding that the mathematical modes had a much higher level of uncertainty than the physical modes. Furthermore, Wu et al. (2020) used the uncertainty in the modal parameters to remove mathematical modes from the stabilization diagram. Therefore, it is of utmost importance to demonstrate that the results of uncertainty quantification are reliable by comparing them with the results obtained from other methods used to estimate uncertainties.

Additionally, estimating the optimal model order of a structure is a difficult task and is often an overestimated parameter leading to the identification of mathematical modes. Stability diagrams are a tool to visualize the identified physical and mathematical modes at different model orders within an interval that aims to separate the physical modes from the mathematical modes. Döhler et al. (2013) modified the procedure proposed by Reynders et al. (2008) to determine more efficiently the covariances of modal parameters in multiple model orders as required by the stabilization diagram.

This paper reports the computation of confidence intervals on the modal parameters identified by using the SSICOV method for two civil structures. Such estimations follow the uncertainty quantification approach proposed by (Reynders et al., 2008) and computationally improved by Döhler et al., (2013). Furthermore, the application of the bootstrap technique, which is a more versatile technique and not restricted to operational modal analysis through the SSI-COV method allows determining the validity of the results. As the bootstrap technique requires repetition of the identification process, a strategy for automatic estimation of modal parameters is configured. The organization of the paper is as follows: i) Section 2.1 introduces the SSI-COV method, ii) Section 2.2 presents the process of automatic estimation of the modal parameters, iii) Sections 3 explain the theory concerning the uncertainty approach from sensitivity analysis and bootstrap approach, and v) Section 4 reports the results of the validation of the two structures.

\section{MODAL PARAMETER IDENTIFICATION.}

This section will describe the procedure used to estimate the vibration modes from the system response. The method used to identify the modal parameters was the SSI-COV method, which is a time-domain parametric method that defines a state-space system realization from the correlation functions of the system response. A stabilization diagram is required in this methodology to identify the modal parameters. The stability diagram is an indicator of the system's poles at different model orders and it is a relatively effective way of identifying modes. However, the results of modal identification can contain spurious modes. Therefore, to extract the physical modes from the stability diagram, in this work, an automatic procedure is configured based on Reynders et al. (2012). This automatic estimation process 
consists of three stages: a) cleaning the stabilization diagram, b) grouping similar modes, and c) selecting the physical modes. The automatic estimation process is applied a priori in the two methodologies used in this work to define the uncertainties of the modal parameters: Uncertainty quantification from (Reynders et al., 2008; Döhler et al., 2013) and Bootstrap technique. Each of process and methodologies applied in this work are described in detail below.

\subsection{COVARIANCE-DRIVEN STOCHASTIC SUBSPACE IDENTIFICATION METHOD (SSI-COV)}

The problem of system realization is as follows: given an external description of a system, determine an internal state variable description that generates the given external description (Antsaklis et al., 2014). The SSI-COV method addresses the stochastic realization problem solved by (Akaike, 1974; Aoki, 1987; Arun et al., 1990), since its objective is the identification from output-only data of the state transition matrix $A \in \mathrm{R}^{\mathrm{n} \times \mathrm{n}}$ and the observation matrix $C \in \mathbb{R}^{r \times n}$ of the following stochastic state-space model:

$$
\begin{aligned}
x_{k+1} & =A x_{k}+w_{k} \\
y_{k} & =C x_{k}+v_{k}
\end{aligned}
$$

where $w_{k}$ and $v_{k}$ are stochastic input processes, which are assumed to have zero mean and a constant spectrum (white noise). From the system matrices $A$ and $C$ the modal parameters can be estimated. The SSI-COV algorithm starts with the evaluation of the output covariance matrices $\Lambda_{l}$ for time lags $l=1,2, \ldots, 2 i-1$. By Assuming ergodicity, these covariance matrices can be formally defined as follows:

$\Lambda_{l}=E\left[y_{k+l} y_{k}^{T}\right]=\lim _{N \rightarrow \infty} \frac{1}{N} \sum_{k=0}^{N-1} y_{k+l} y_{k}^{T}$

Since a finite number of samples $N$ is available in practical applications, an estimation $\Lambda_{l}$ can be obtained by dropping the limit. However, the direct use of this expression can be very time-consuming; hence, another alternative consists in using an approach based on the Fast Fourier Transform (FFT) (Oppenheim et al., 1975). In a second step, these output covariance matrices are organized in a block Toeplitz matrix $T_{1 \mid i} \in \mathbb{R}^{\text {ir } \times i r}$ :

$T_{1 \mid i}=\left[\begin{array}{cccc}{\left[\Lambda_{i}\right]} & {\left[\Lambda_{i-1}\right]} & \cdots & {\left[\Lambda_{1}\right]} \\ {\left[\Lambda_{i+1}\right]} & {\left[\Lambda_{i}\right]} & \cdots & {\left[\Lambda_{2}\right]} \\ \cdots & \cdots & \cdots & \cdots \\ {\left[\Lambda_{2 i-1}\right]} & {\left[\Lambda_{2 i-2}\right]} & \cdots & {\left[\Lambda_{i}\right]}\end{array}\right]$,

where $i$ is the number of block rows of the Toeplitz matrix and $r$ the total number of sensors. Furthermore, one of the properties for stochastic state-space models is the possibility to decompose the output covariance matrices in the form: $\Lambda_{l}=C A^{l-1} G$ where $G \in \mathbb{R}^{r \times n}$ is the next-state output covariance matrix given by $G=E\left[x_{k+1} y_{k}^{T}\right]$. According to these properties, $T_{1 \mid i}$ can be decomposed as shown in Equation (4), where $\mathcal{O} \in \mathbb{R}^{i r \times n}$ is the extended observability matrix, $\Gamma \in$ $\mathbb{R}^{n \times i r}$ the reversed extended stochastic controllability matrix, and $n$ the model order.

$T_{1 \mid i}=\mathcal{O} \Gamma=\left[\begin{array}{c}C \\ C A \\ \cdots \\ C A^{i-1}\end{array}\right]\left[\begin{array}{llll}A^{i-1} G & \cdots & A G & G\end{array}\right]$.

This Equation shows that $T_{1 i}$ is the result of multiplying a matrix with $n$ columns by a matrix with $n$ rows. Then, for ir $\geq n$ and an observable and controllable system, the rank of the $i r$ by $i r$ Toeplitz matrix is $n$. The application of the singular value decomposition (SVD) allows rewriting $T_{1 \mid i}$ as:

$T_{1 \mid i}=U S V^{T}=\left[\begin{array}{ll}U_{1} & U_{2}\end{array}\right]\left[\begin{array}{cc}S_{1} & 0 \\ 0 & S_{2}\end{array}\right]\left[\begin{array}{l}V_{1} \\ V_{2}\end{array}\right]^{T}=U_{1} S_{1} V_{1}^{T}$

where $U \in \mathbb{R}^{i r \times i r}$ and $V \in \mathbb{R}^{i r \times i r}$ are orthonormal matrices, with $U^{T} U=U U^{T}=I \quad \in \quad \mathbb{R}^{i r \times i r}$ and $V^{T} V=V V^{T}=I \in$ $\mathbb{R}^{i r \times i r} . S \in \mathbb{R}^{i r \times i r}$ is a diagonal matrix containing the positive singular values in descending order. The observability and controllability matrices can be determined from the reduced singular value decomposition as shown in Equation (6), 
where $S_{1} \in \mathbb{R}^{n \times n}$ contains the non-zero singular values with $U_{1} \in \mathbb{R}^{i r \times n}$ and $V_{1} \in \mathbb{R}^{i r \times n}$ their corresponding singular vectors.

$\mathcal{O}=U_{1} S_{1}^{1 / 2}$

$\Gamma=S_{1}^{1 / 2} V_{1}^{T}$

Identifying system matrices $A$ and $C$ is simple from the previously computed observability $\mathcal{O}$ and $\Gamma$ controllability matrices. $C$ equals the first $r$ rows of $\mathcal{O}$, and the state transition matrix $A$ can be estimated by exploiting the shift structure of $\mathcal{O}$ (Kung, 1978), as follows:

$$
\left[\begin{array}{c}
C A \\
C A^{2} \\
\cdots \\
C A^{i}
\end{array}\right]=\left[\begin{array}{c}
C \\
C A \\
\cdots \\
C A^{i-1}
\end{array}\right] A \Leftrightarrow A=\left[\begin{array}{c}
C \\
C A \\
\cdots \\
C A^{i-1}
\end{array}\right]^{\dagger}\left[\begin{array}{c}
C A \\
C A^{2} \\
\cdots \\
C A^{i}
\end{array}\right]=\underline{\mathcal{O}}^{\dagger} \overline{\mathcal{O}}
$$

where $(\cdot)^{\dagger}$ represents the Moore-Penrose pseudo-inverse of a matrix. $\mathcal{O}$ contains the first $r(i-1)$ rows of $\mathcal{O}$, while $\overline{\mathcal{O}}$ contains the last $r(i-1)$ rows of $\mathcal{O}$. Alternatively, the matrix $A$ could also be computed from the decomposition property of a shifted block Toeplitz matrix (Zeiger et al., 1974):

$T_{2 \mid i+1}=\mathcal{O} A \Gamma$

where the shifted matrix $T_{2 \mid i+1}$ is composed of the output covariance matrices $\Lambda_{l}$ for time lags $l=2,3, \ldots, 2 i$. Introducing Equation (6) into Equation (8) and solving for $A$ gives:

$A=\mathcal{O}^{\dagger} T_{2 \mid i+1}(\Gamma)^{\dagger}=S_{1}^{-1 / 2} U_{1}^{T} T_{2 \mid i+1} V_{1} S_{1}^{-1 / 2}$

From matrices $A$ and $C$ the modal parameters are extracted. The decomposition of the state transition matrix $A$ is given by:

$A=\Psi \Lambda \Psi^{-1}, \quad A \psi_{k}=\lambda_{k} \psi_{k}$

where $\psi_{k}$ and $\lambda_{k}$ are the discrete-time eigenvectors and eigenvalues, respectively. As $A$ was obtained from the discretization of the continuous-time matrix $A_{c}$, the equivalent continuous-time eigen-properties can be computed as follows:

$\psi_{c k}=\psi_{k}, \quad \lambda_{c k}=\frac{\ln \left(\lambda_{k}\right)}{\Delta t}$

Finally, natural frequencies and damping ratios are computed from:

$f_{k}=\frac{\left|\lambda_{c k}\right|}{2 \pi} \quad[H z]$

$\xi_{k}=-\frac{\Re\left(\lambda_{c k}\right)}{\left|\lambda_{c k}\right|} * 100 \quad[\%]$

where $\mathfrak{R}(\bullet)$ represents the real part of a complex number. Finally, the observed mode shapes $\phi_{k}$ can be computed through the multiplication of matrix $C$ with eigenvectors of matrix $A$ :

$\phi_{k}=C \psi_{k}$. 


\subsection{AUTOMATIC MODAL PARAMETER ESTIMATION (MPE)}

An automated procedure based on the methodology proposed in Reynders et al. (2012) is configured to extract the physical modes obtained with the SSI-COV method. The automation of the MPE consists of three stages: cleaning the stabilization diagram, grouping similar modes, and selecting the physical modes.

a) Cleaning the estabilization diagram.

Hard validation criteria (HVC) and soft validation criteria (SVC) are used for mode elimination in the cleaning stage. The HVC are applied to all modes. Modes that do not meet the following criteria are eliminated immediately: i) $\xi_{k}>0 \%$ and $\xi_{k}<10 \%$, ii) $f_{k}<f_{s} / 2$, where $f_{s}$ is the sampling frequency, and iii) mode with a complex conjugate pair. The SVC are summarized in Table 1. A mode is composed of a frequency $(f)$, a damping ratio $(\xi)$, and a mode shape $(\phi)$, then, assuming that $i$ and $j$ are a pair of closest modes according to the distance:

$d(i, j)=d_{f}\left(f_{i}, f_{j}\right)+1-\operatorname{MAC}\left(\phi_{i}, \phi_{j}\right)$,

$d_{f}, d_{\xi}$ and $M A C$ measure the similarity between these modes. On the other hand, the $M P C$ and $M P D$ indicators measure the complexity of the mode shapes. (Reynders et al., 2012), presents detailed information on how to compute these criteria.

Table 1 Soft validation criteria (SVC).

\begin{tabular}{cccc}
\hline Criterion & Value & Ideal Physical & Ideal Mathematical \\
\hline$\zeta^{(1)}$ & $d_{f}=$ Relative frequency & 0 & 1 \\
$\zeta^{(2)}$ & $d_{\xi}=$ Relative damping ratio & 0 & 1 \\
$\zeta^{(3)}$ & $M A C=$ Modal Assurance & 1 & 0 \\
$\zeta^{(4)}$ & $M P C=$ Modal Phase Collinearity & 1 & 0 \\
$\zeta^{(5)}$ & $M P D=$ Mean Phase Deviation & 0 & 1 \\
\hline
\end{tabular}

Based on these discriminative criteria, an iterative Fuzzy C-means (FCM) clustering algorithm is employed to classify the modes into likely physical and mathematical modes. This approach divides a dataset into $\mathrm{C}$ clusters or classes by minimizing the weighted sum of the Euclidean distance between the dataset and centroids of each class. Thus, for the case in question, the objective function of the FCM can be written as:

$J_{m}=\sum_{j=1}^{n_{p}} \sum_{i=1}^{2} \eta_{i j}^{m}\left\|\zeta_{j}-V_{i}\right\|^{2}$,

where $\eta_{i j}$ is the degree of membership of the mode $j$ to the class $i$ (likely physical mode or mathematical mode), $V_{i}$ is the center of the classes, and $m \geq 1$ (commonly set to 2 ) is a weighting exponent. The objective function optimization is carried out through an iterative process, updating each class's degrees of membership and centroids until a termination criterion is met, either a maximum number of iterations or a minimum error. In fuzzy logic theory, the degree of membership represents a value in the range of 0 and 1 . A value closer to 1 indicates that an element belongs to a particular class, while values closer to 0 indicate that the element does not belong to the particular class. As a result, the factor $0<\eta_{i j}<1$ indicates how much the modes $j=1,2, \ldots, n_{p}$ belong to each class $i=1,2$. As the summation of membership of each mode should be equal to one, modes with a degree of membership of less than 0.5 to the probably physical class are considered mathematical modes and are eliminated.

b) Grouping similar modes

After the cleaning stage, the probably physical modes are grouped according to similar features. This grouping is carried out using a hierarchical clustering approach in which the similarity information between each pair of modes computed through Equation (15) is used to construct a distance matrix $D$. Then, modes/clusters that are in proximity are linked together using a linkage function. Finally, using a threshold $d_{h}$, cut the hierarchical tree into clusters. In 
(Reynders et al., 2012), it is assumed that the likely physical modes are normally distributed, and the following threshold is proposed:

$d_{h}=\mu_{p}+2 \sigma_{p}$

where $\mu_{p}$ and $\sigma_{p}$ are the mean and standard deviation, respectively, of $\zeta_{i}^{(1)}+1-\zeta_{i}^{(3)}$ for $i=1,2, \ldots, n_{e}$, where $\zeta_{i}^{(1)}$ and $\zeta_{i}^{(3)}$ represent the criteria $d_{f}$ and $M A C$, respectively, in Table 1 and $n_{e}$ represents the number of likely physical modes.

Not all mathematical modes will likely be eliminated in the cleaning stage; therefore, the set of clusters obtained in the clustering phase can be divided into two classes: those containing physical modes and those having mathematical or spurious modes. Generally, physical modes are consistently identified in each model order from a specific model order on, while mathematical modes do not appear with such consistency. For this reason, clusters that contain physical modes are expected to have more elements than those that include mathematical modes. In this research, the FCM algorithm is used to select the clusters containing the physical modes. In this case, the objective function to minimize is

$J_{m}=\sum_{j=1}^{n_{c}} \sum_{i=1}^{2} \eta_{i j}^{m}\left(\mathcal{L}_{j}-\mathrm{V}_{i}\right)^{2}$,

where $\mathcal{L}_{j}$ is the number of elements of the cluster $j, n_{c}$ is the number of clusters, $\eta_{i j}$ is the degree of membership of the cluster $j$ to the class (physical or mathematical), $V_{i}$ is the center of classes, and $m$ is the weighting exponent Clusters with a degree of membership of higher than 0.5 to the physical class are selected as the final clusters.

c) Selecting the physical modes

Finally, the automatic MPE ends with the selection of the representative mode for each cluster. In this work, the following strategy is proposed. First, in the grouping stage, a distance matrix $D \in \mathbb{R}^{n_{e} \times n_{e}}$ was constructed, then $D^{j} \in \mathbb{R}^{\mathcal{L}_{j} \times \mathcal{L}_{j}}$ is a subset of this matrix containing the distances of the elements of the cluster $j$. The smallest element of $s^{j} \in \mathbb{R}^{\mathcal{L}_{j}}$ in Equation (19) corresponds to the $k$ mode, which represents the cluster $j$. In this way, the mode closest to all modes, and with a high $M P C$ value is selected as representative.

$s^{j}=\left(\sum_{i=1}^{\mathcal{L}_{j}} D_{i k}^{j}\right)+1-M P C_{k}$.

\section{UNCERTAINTIES OF MODAL PARAMETERS}

\subsection{Approach 1: Uncertainty quantification from (Reynders et al., 2008; Döhler et al., 2013)}

The uncertainties of modal parameters can be quantified through statistical values such as the variance or its square root, standard deviation. (Reynders et al., 2008) proposed a procedure to determine these uncertainties at a given model order by the propagation of perturbations of the collected data in each SSI-COV stage. Thus, the covariance of the modal parameters depends on the covariance of the system matrices, which depends on the covariance of the Toeplitz matrix.

The quantification of the uncertainties in the system matrices is performed in two steps. In the first step, the uncertainty $\Delta T_{1 \mid i}$ of the Toeplitz matrix is propagated to the uncertainty $\Delta \mathcal{O}$ of the observability matrix through the Jacobian matrix $J_{\mathcal{O}, T_{1 \mid i}}$. In the second step, the uncertainty $\Delta \mathcal{O}$ of the observability matrix is propagated to the uncertainty $\Delta A$ and $\Delta C$ of the system matrices through the Jacobian matrices $J_{A, \mathcal{O}}$ and $J_{C, \mathcal{O}}$, respectively. Mathematically, it is expressed as:

$\operatorname{vec}(\Delta \mathcal{O})=J_{\mathcal{O}, T_{1 \mid i}} \operatorname{vec}\left(\Delta T_{1 \mid i}\right)$

$\left[\begin{array}{l}\operatorname{vec}(\Delta A) \\ \operatorname{vec}(\Delta C)\end{array}\right]=\left[\begin{array}{l}J_{A, \mathcal{O}} \\ J_{C, \mathcal{O}}\end{array}\right] \operatorname{vec}(\Delta \mathcal{O})$

where $\operatorname{vec}(\bullet)$ is the stacking vectorization operator. The covariance of the system matrices is then computed as: 
$\operatorname{cov}\left(\left[\begin{array}{l}\operatorname{vec}(A) \\ \operatorname{vec}(C)\end{array}\right]\right)=\left[\begin{array}{l}J_{A, \mathcal{O}} \\ J_{C, \mathcal{O}}\end{array}\right] J_{\mathcal{O}, T_{1 \mid i}} \operatorname{cov}\left(\operatorname{vec}\left(T_{1 \mid i}\right)\right) J_{\mathcal{O}, T_{1 \mid i}}{ }^{T}\left[\begin{array}{c}J_{A, \mathcal{O}} \\ J_{C, \mathcal{O}}\end{array}\right]^{T}$

The uncertainties $\Delta f_{k}, \Delta \xi_{k}$ and $\Delta \varphi_{k}$ of the modal parameters (natural frequencies $f_{k}$, damping ratios $\xi_{k}$ and modal shapes $\varphi_{k}$ ) are connected to the uncertainties $\Delta A$ and $\Delta C$ of the system matrices through:

$\left[\begin{array}{l}\Delta f_{k} \\ \Delta \xi_{k}\end{array}\right]=\left[\begin{array}{l}J_{f_{k}, A} \\ J_{\xi_{k}, \mathrm{~A}}\end{array}\right] \operatorname{vec}(\Delta A)$

$\Delta \varphi_{k}=J_{\varphi_{k}, A, C} \operatorname{vec}\left[\begin{array}{l}\Delta A \\ \Delta C\end{array}\right]$

where $J_{f_{k}, A}, J_{\xi_{k}, \mathrm{~A}}$, and $J_{\varphi_{k}, A, C}$ are the Jacobians for each mode $k$. Finally, the covariance of the modal parameters are estimated as:

$\operatorname{cov}\left(\left[\begin{array}{l}f_{k} \\ \xi_{k}\end{array}\right]\right)=\left[\begin{array}{c}J_{f_{k}, A} \\ J_{\xi_{k}, \mathrm{~A}}\end{array}\right] \underbrace{J_{A, \mathcal{O}} J_{\mathcal{O}, T_{1 \mid i}} \operatorname{cov}\left(\operatorname{vec}\left(T_{1 \mid i}\right)\right) J_{A, \mathcal{O}}{ }^{T} J_{\mathcal{O}, T_{1 \mid i}}{ }^{T}}_{\operatorname{cov}(\operatorname{vec}(A))}\left[\begin{array}{c}J_{f_{k}, A} \\ \left.\xi_{\xi_{k}, \mathrm{~A}}\right]^{T}\end{array}\right.$

$\operatorname{cov}\left[\begin{array}{c}\Re\left(\varphi_{k}\right) \\ \mathfrak{I}\left(\varphi_{k}\right)\end{array}\right]=\left[\begin{array}{c}\Re\left(J_{\varphi_{k}, A, C}\right) \\ \mathfrak{I}\left(J_{\varphi_{k}, A, C}\right)\end{array}\right] \underbrace{\left[\begin{array}{l}J_{A, \mathcal{O}} \\ J_{C, \mathcal{O}}\end{array}\right] J_{\mathcal{O}, T_{1 \mid i}} \operatorname{cov}\left(\operatorname{vec}\left(T_{1 \mid i}\right)\right) J_{\mathcal{O}, T_{1 \mid i}}{ }^{T}\left[\begin{array}{c}J_{A, \mathcal{O}} \\ J_{C, \mathcal{O}}\end{array}\right]^{T}}_{\operatorname{cov}\left(\left[\begin{array}{l}\operatorname{vec}(A) \\ \operatorname{vec}(C)\end{array}\right]\right)}\left[\begin{array}{c}\Re\left(J_{\varphi_{k}, A, C}\right) \\ \mathfrak{I}\left(J_{\varphi_{k}, A, C}\right)\end{array}\right]^{T}$

To determine the confidence intervals of a mode $k$ requires the availability of a large data sample, which requires a high computational cost. In this method, the original acceleration time series is divided into $n_{b}$ statistically independent blocks of length $N_{b}$ such that $n_{b} N_{b}=N$, where $N$ is the total number of data used to compute the output covariance matrices with Equation (2). Then, from this division, estimates of the Toeplitz matrix are calculated, and subsequently, with these estimates, the covariance $\operatorname{cov}\left(\operatorname{vec}\left(T_{1 \mid i}\right)\right)$ is calculated. This allows the determination of confidence intervals on modal parameters in a given order without repeating the identification process for each data block. However, the computational cost of the direct application of this method can also be expensive for high model orders and large sensor networks. Therefore, (Döhler et al. 2013) reformulated the procedure mathematically and proposed an algorithm to be efficiently applied to calculate the covariance of all modes in the stability diagram. The computational cost reduction was achieved by calculating the uncertainty bounds at the maximum-desired order before the calculations of the other orders. In this paper, this algorithm is implemented and applied immediately after obtaining the modes with the SSI-COV method. Then, the automatic modal parameter estimation procedure described in the previous section extracts the physical modes with their respective standard deviations.

\subsection{Approach 2: Bootstrap technique}

The bootstrap method was developed by (Efron, 1979). Suppose the random variable $y$ is the outcome of some stochastic process with unknown probability distribution $F$, and $n$ independent measurements collected in the sample $Y=\left\{y_{1}, y_{2}, \ldots, y_{n}\right\}$ are available to estimate a parameter of interest $s(Y)$. The bootstrap method creates additional data collections, denoted $Y^{(b) *}=\left\{y_{1}^{*(b)}, y_{2}^{*(b)}, \ldots, y_{n}^{*(b)}\right\}$, as a randomized or resampled version of the original sample $Y=\left\{y_{1}, y_{2}, \ldots, y_{n}\right\}$. Once these additional samples are formed, the usual sample statistics can be applied. The basic assumptions in this method are: The measured outcomes of the random variable, and collected in $Y=\left\{y_{1}, y_{2}, \ldots, y_{n}\right\}$, must be independent, and the measured outcomes $y_{i}$ must be representative of the random source. When working with time series, the first assumption is violated because $Y=\left\{y_{1}, y_{2}, \ldots, y_{n}\right\}$ is a collection of serially dependent measurements. Hence, applying the resampling process with individual outcomes will break up the covariance structure of the time series. Then, it is possible to extend the method to time series through the resample blocks of data rather than individual observations. Thus, the moving blocks bootstrap are a simple resampling algorithm used for time series.

The steps to apply the moving block bootstrap given by (Kunsch, 1979) can be summarized as follows: 
1. Break the time series $Y=\left\{y_{1}, y_{2}, \ldots, y_{n}\right\}$, into $n-l+1$ overlapping blocks $B_{i}=\left\{y_{i}, y_{i+1}, \ldots, y_{i+l-1}\right\}$ of length $l$ for $i=1,2, \ldots, n-l+1$. Then, form the collection $B=\left\{B_{1}, B_{2}, \ldots, B_{n-l+1}\right\}$.

2. Resample $k=n / l$ blocks $B_{i}$ with replacement to form $B$ bootstrap time series replica by collecting the $k$ resampled $B_{i}$. For example, the $b_{t h}$ bootstrapped time series may be: $Y^{(b) *}=\left\{B_{1}, B_{5}, \ldots, B_{n-l+1}, \ldots, B_{1}\right\}$.

3. Compute the bootstrap replica of the statistic of interest, $s\left(Y^{(b) *}\right)$. For instance, this estimator could be the sample correlation estimates $\Lambda_{l}^{Y(b) *}$ of the bootstrapped time series $Y^{(b) *}$, where $l$ denotes the lag.

4. Compute the sample statistic of interest over the ensemble of the $B$ generated bootstrap replica $s\left(Y^{(b) *}\right)$.

In Figure 1. a time series with length $n=12$ and block length equal to $l=3$ illustrate steps 1 and 2 schematically.

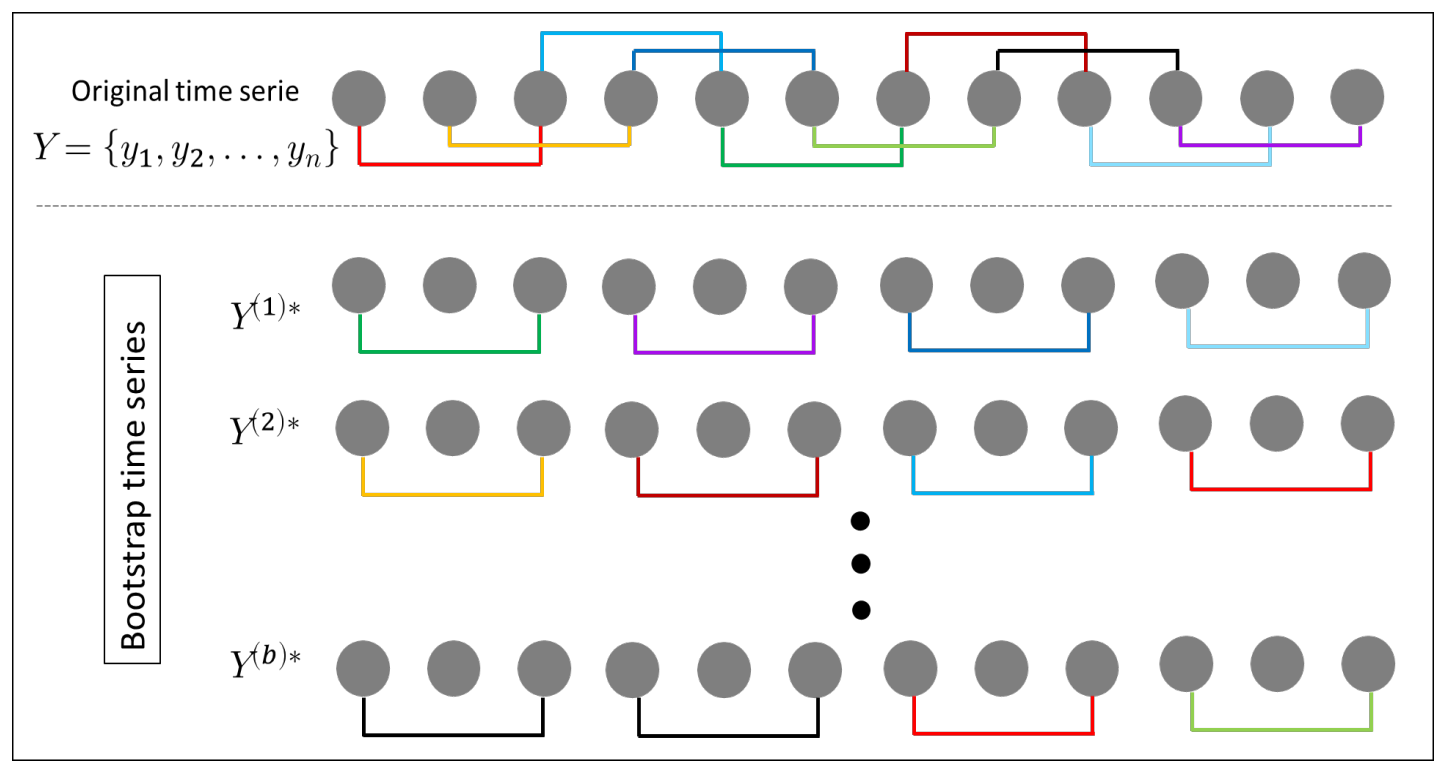

Figure 1. Moving block bootstrap: schematic diagram.

Bootstrap for operational modal analysis

As mentioned in the previous section, to obtain modal parameters statistics such as mean, standard deviation, and confidence intervals, it is necessary to have a sufficiently large set of modal parameters. In addition to repeating the identification process B times, this involves performing B measurement tests to obtain B time series long enough for acceptable identification. Furthermore, calculating the uncertainty in the modal parameters obtained from ambient vibrations measured at different time instants requires a preliminary analysis to identify whether environmental and operational variables influence the modal parameters. Therefore, these limitations make the process of repeating the measurement tests impractical. The Bootstrap technique allows the simulation of response data from only the set of available measurements. Thus, during the application of the Bootstrap method, it is assumed that there is only one set of simultaneous response data measured at different locations along with the structure.

According to Giampellegrini (2007), once response data are obtained, it is then possible to get a collection of B sets of bootstrapped modal parameters. Therefore, in this paper, for each simulated response, the SSI-COV method is applied. In this way, a set B of stabilization diagrams is obtained. Subsequently, the automated modal parameter estimation process extracts the physical modes from each of these diagrams. These physical modes are compared using the physical modes obtained with the original time series through the relative distance between the natural frequencies to group the similar modes. As a mode $k$ may not be identified in each repetition, the whole procedure is performed until for each physical mode; there is a set $B^{*}$ of modal parameters, which the statistics of the system's model can be determined. Finally, the $95 \%$ confidence intervals for each vibration mode are estimated as the $\pm 2 \sigma$ bound.

\section{VALIDATION OF UNCERTAINTY BOUNDS}

\subsection{Validation with simulated data}

A simulation of experimental data was performed to obtain data similar to those obtained in a dynamic test of ambient vibration measurement. The structural model consists of a simply supported beam. The structure has a length 
of $10[\mathrm{~m}]$, a uniform square cross-section equal to $0.2[\mathrm{~m}]$, and is discretized 11 elements of equal length. The Young's modulus in each element is $E=1.787 * 10^{9}\left[\mathrm{~N} / \mathrm{m}^{2}\right]$ and density is equal to $12816 \mathrm{~kg} / \mathrm{m}^{3}$. These properties are just generic and do not belong to any material. As shown in Figure 2, this beam is also elastically restrained against translational and rotational movements at nodes B and C. $k_{T}=440000[\mathrm{~N} / \mathrm{m}]$ and $k_{R}=880000[\mathrm{~N} / \mathrm{m}]$ are the translational spring and rotational spring constants, respectively.

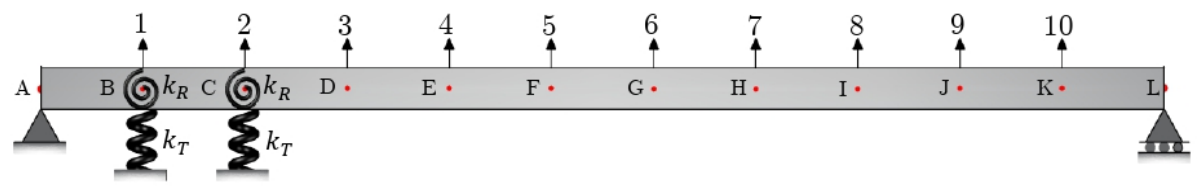

Figure 2. Structural model.

The mass and stiffness matrices were obtained from the geometric and material properties and condensed into ten vertical degrees of freedom to reduce the problem size. Then, vertical accelerations time series with durations of $15[\mathrm{~min}]$ at a sampling frequency of $200[\mathrm{~Hz}]$ were simulated, considering a noise level of $S N R=40[\mathrm{~dB}]$. All ten vertical degrees of freedom were loaded with gaussian excitation loads. These simulated data were used for the identification of modal parameters using the SSI-COV method. The number of block rows of the Toeplitz matrix $T_{1 i}$ was set in $i=60$, and for constructing the stability diagram, a range of orders from $n=2$ to $n=120$ in increments of 2 was used. Then, the approach 1 to quantifying uncertainties was applied with a number of blocks $n_{b}=100$. As the entire series consists of $N=180000$, each block comprises $N_{b}=1800$ samples, which amounts to $9 s$ of data per block.

The automated MPE procedure was implemented to extract the physical modes of the structure. Figure 3. (a) shows the stabilization diagram constructed immediately after the cleaning stage. The stabilization diagram shows the poles identified in the system by the SSI-COV method, however performing a variation of the model order of the state space matrix A. Thus, this diagram allows observing stable poles of the system as the model order is varied. Then, in Figure 3 , the classification of the poles into the probably physical and mathematical groups can be observed when applied the cleaning stage. The horizontal bars indicate the standard deviation of each pole. According to these results, there are both probably physical and mathematical poles with high uncertainties. However, in Figure 3.(b) shows the stability diagram constructed after applying the automated MPE procedure, it can be seen that the modes selected as physical (blue vertical lines) present a lower uncertainty, as the standard deviations are imperceptible. According to the work of Verboven et al (2004), it is concluded that the uncertainty levels of the physical modes are much smaller than for the mathematical modes. Thus, the obtained results support these conclusions and validated the standard deviations' consistency computed through approach 1. In the stabilization diagram of Figure $3(\mathrm{~b})$, the red dots are the representative modes of each cluster, and in Figure 4, the extracted information is shown. It can be observed in the polar plot that all representative modes are monophasic, thus representing real modes.

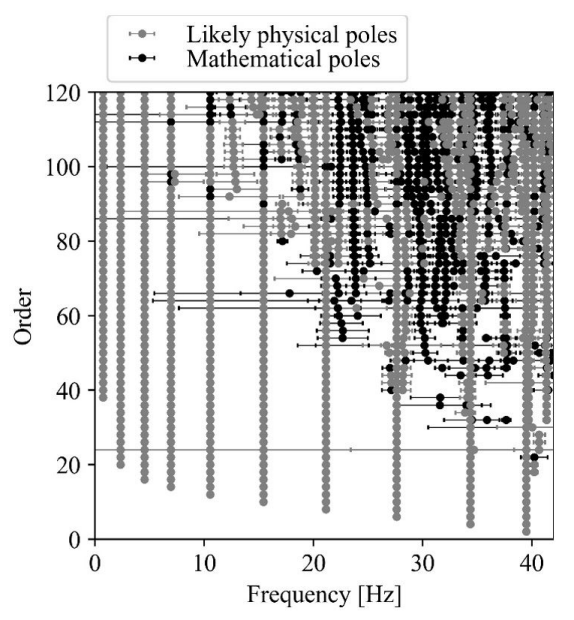

(a)

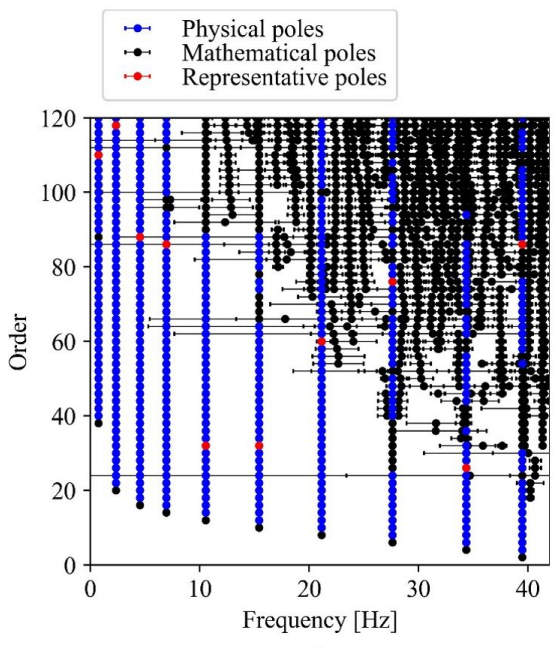

(b)

Figure 3. Example 1: (a) Stability diagram after the cleaning stage. (b) Stability diagram after the MPE procedure. 
For the bootstrap, 1000 pseudo time series were generated through the moving blocks resampling method with either length $l=30000$ samples or $150 \mathrm{~s}$. Tables 2 and 3 and Figure 5 present the results of the $\pm 2 \sigma$ uncertainty bounds estimated using the two approaches for frequencies, damping ratios, and vibration modes, respectively. The means obtained for the three modal parameters (frequencies, damping ratios, mode shapes) are pretty close to the parameters shown in Figure 4. Thus, the bias errors found are minor, indicating that the bootstrap result is robust. The standard deviations estimated using the two approaches are similar in magnitude. Regarding the confidence intervals, these differ a little for the two approaches. However, they overlap, so it is verified that approach 1 provides consistent results concerning the Bootstrap approach. Figures 6 and 7 show the cumulative distributions of the bootstrap samples obtained for the natural frequencies and damping ratios. It is observed that the identified modes are normally distributed in all cases.

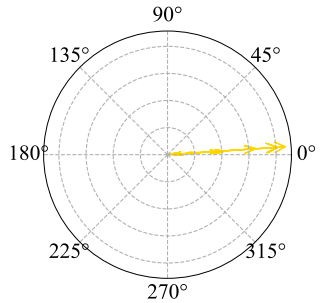

Mode 1

$f_{1}=0.727 \mathrm{~Hz}$

$\xi_{1}=\mathbf{5 . 8 5} \%$

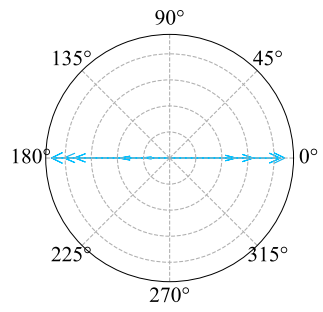

Mode 6

$f_{6}=15.422 \mathrm{~Hz}$

$\xi_{6}=0.256 \%$

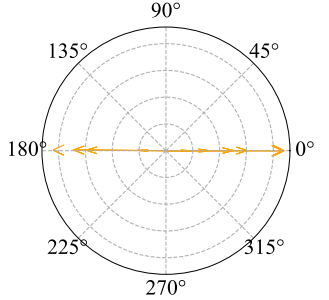

Mode 2

$f_{2}=2.336 \mathrm{~Hz}$

$$
\xi_{2}=1.699 \%
$$

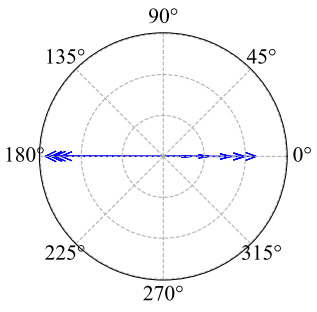

Mode 7

$f_{7}=21.115 \mathrm{~Hz}$

$\xi_{7}=0.180 \%$

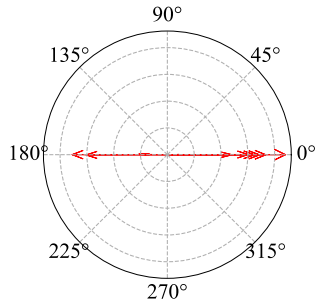

Mode 3

$f_{3}=4.505 \mathrm{~Hz}$

$\xi_{3}=0.837 \%$

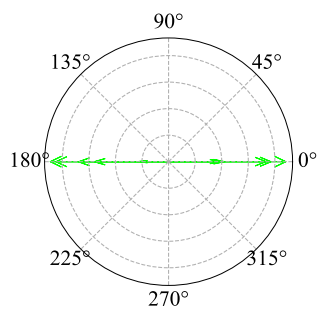

Mode 8

$f_{8}=27.6 \mathrm{~Hz}$

$\xi_{8}=0.122 \%$

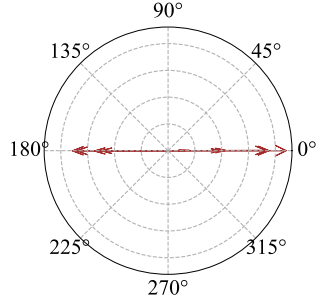

Mode 4

$f_{4}=6.923 \mathrm{~Hz}$

$\xi_{4}=0.544 \%$

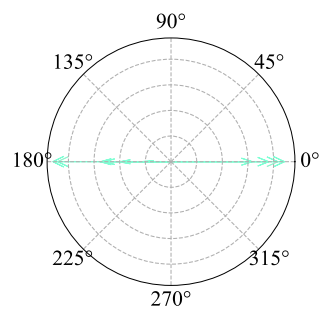

Mode 9

$f_{9}=34.358 \mathrm{~Hz}$

$\xi_{9}=0.101 \%$

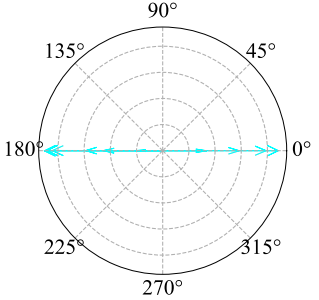

Mode 5

$f_{5}=10.528 \mathrm{~Hz}$

$\xi_{5}=\mathbf{0 . 3 8 5} \%$

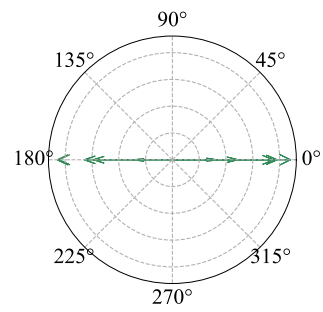

Mode 10

$f_{10}=39.466 \mathrm{~Hz}$

$\xi_{10}=0.084 \%$

Figure 4. Modal parameters identified in the structural model.

Table 2. Example 1: Confidence intervals of natural frequencies.

\begin{tabular}{|c|c|c|c|c|c|c|c|c|}
\hline \multirow{3}{*}{ Mode } & \multicolumn{5}{|c|}{ Bootstrap verification } & \multicolumn{3}{|c|}{$\begin{array}{l}\text { Uncertainty quantification from } \\
\text { (Reynders et al., 2008; Döhler et al., 2013) }\end{array}$} \\
\hline & \multirow{2}{*}{ Estimate } & \multirow{2}{*}{ Bias } & \multirow{2}{*}{$\begin{array}{l}\text { Standard } \\
\text { Deviation }\end{array}$} & \multicolumn{2}{|c|}{ 95\% Confidence intervals } & \multirow{2}{*}{$\begin{array}{l}\text { Standard } \\
\text { Deviation }\end{array}$} & \multicolumn{2}{|c|}{ 95\% Confidence intervals } \\
\hline & & & & Lower & Upper & & Lower & Upper \\
\hline 1 & 0.7187 & 8.17E-03 & 0.0248 & 0.6691 & 0.7683 & 0.0260 & 0.6749 & 0.7789 \\
\hline 2 & 2.3358 & $8.18 \mathrm{E}-05$ & 0.0017 & 2.3324 & 2.3392 & 0.0029 & 2.3301 & 2.3417 \\
\hline 3 & 4.5056 & $2.09 \mathrm{E}-04$ & 0.0024 & 4.5008 & 4.5105 & 0.0025 & 4.5004 & 4.5104 \\
\hline 4 & 6.9228 & $6.63 \mathrm{E}-05$ & 0.0017 & 6.9194 & 6.9261 & 0.0025 & 6.9178 & 6.9278 \\
\hline 5 & 10.5283 & $1.35 \mathrm{E}-04$ & 0.0021 & 10.5241 & 10.5325 & 0.0025 & 10.5234 & 10.5334 \\
\hline 6 & 15.4208 & $9.51 \mathrm{E}-04$ & 0.0017 & 15.4174 & 15.4243 & 0.0020 & 15.4178 & 15.4258 \\
\hline 7 & 21.1160 & $8.00 \mathrm{E}-04$ & 0.0016 & 21.1128 & 21.1192 & 0.0021 & 21.1110 & 21.1194 \\
\hline 8 & 27.6007 & $7.95 \mathrm{E}-04$ & 0.0022 & 27.5964 & 27.6050 & 0.0033 & 27.5933 & 27.6065 \\
\hline 9 & 34.3577 & $1.80 \mathrm{E}-04$ & 0.0027 & 34.3523 & 34.3631 & 0.0022 & 34.3531 & 34.3619 \\
\hline 10 & 39.4666 & $5.72 \mathrm{E}-04$ & 0.0024 & 39.4618 & 39.4714 & 0.0052 & 39.4556 & 39.4764 \\
\hline
\end{tabular}


Table 3. Example 1: Confidence intervals of damping ratios.

\begin{tabular}{|c|c|c|c|c|c|c|c|c|}
\hline \multirow{3}{*}{ Mode } & \multicolumn{5}{|c|}{ Bootstrap verification } & \multicolumn{3}{|c|}{$\begin{array}{c}\text { Uncertainty quantification from } \\
\text { (Reynders et al., 2008; Döhler et al., 2013) }\end{array}$} \\
\hline & \multirow{2}{*}{ Estimate } & \multirow{2}{*}{ Bias } & \multirow{2}{*}{$\begin{array}{l}\text { Standard } \\
\text { Deviation }\end{array}$} & \multicolumn{2}{|c|}{ 95\% Confidence intervals } & \multirow{2}{*}{$\begin{array}{l}\text { Standard } \\
\text { Deviation }\end{array}$} & \multicolumn{2}{|c|}{ 95\% Confidence intervals } \\
\hline & & & & Lower & Upper & & Lower & Upper \\
\hline 1 & 6.1800 & $3.28 \mathrm{E}-01$ & 0.7480 & 4.6840 & 7.6760 & 0.7590 & 4.3335 & 7.3695 \\
\hline 2 & 1.6583 & $4.10 \mathrm{E}-02$ & 0.1382 & 1.3819 & 1.9347 & 0.1060 & 1.4873 & 1.9113 \\
\hline 3 & 0.8555 & $1.86 \mathrm{E}-02$ & 0.0443 & 0.7669 & 0.9441 & 0.0446 & 0.7478 & 0.9262 \\
\hline 4 & 0.5443 & $1.40 \mathrm{E}-04$ & 0.0355 & 0.4733 & 0.6154 & 0.0257 & 0.4931 & 0.5959 \\
\hline 5 & 0.3898 & $5.22 \mathrm{E}-03$ & 0.0252 & 0.3394 & 0.4402 & 0.0177 & 0.3492 & 0.4200 \\
\hline 6 & 0.2601 & $3.96 \mathrm{E}-03$ & 0.0147 & 0.2307 & 0.2895 & 0.0143 & 0.2275 & 0.2847 \\
\hline 7 & 0.1899 & $1.04 \mathrm{E}-02$ & 0.0141 & 0.1618 & 0.2180 & 0.0086 & 0.1623 & 0.1967 \\
\hline 8 & 0.1250 & 3.37E-03 & 0.0063 & 0.1123 & 0.1377 & 0.0082 & 0.1052 & 0.1380 \\
\hline 9 & 0.1022 & $7.49 \mathrm{E}-04$ & 0.0127 & 0.0768 & 0.1277 & 0.0050 & 0.0915 & 0.1115 \\
\hline 10 & 0.0930 & 8.81E-03 & 0.0087 & 0.0756 & 0.1104 & 0.0114 & 0.0614 & 0.1070 \\
\hline
\end{tabular}
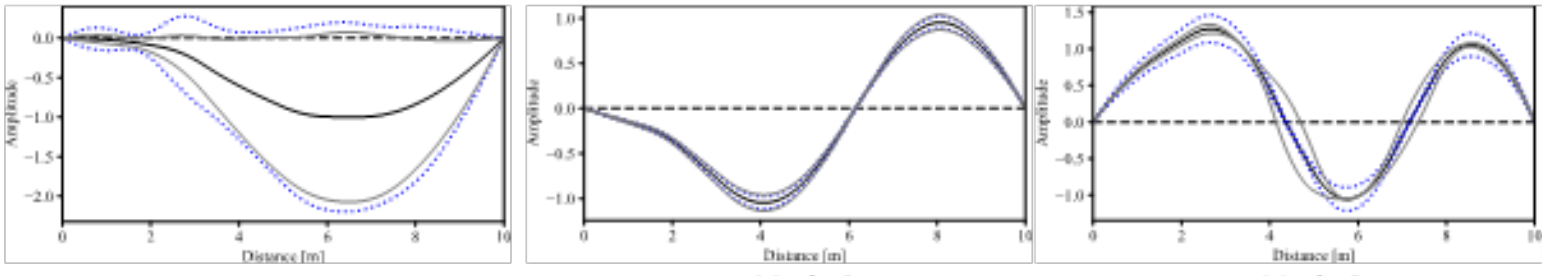

\section{Mode 1}

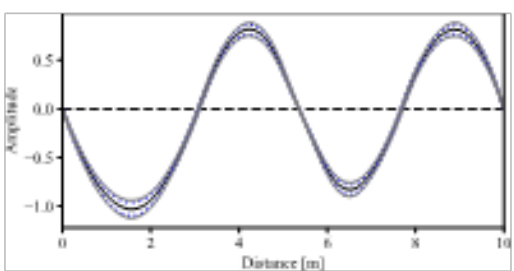

Mode 2
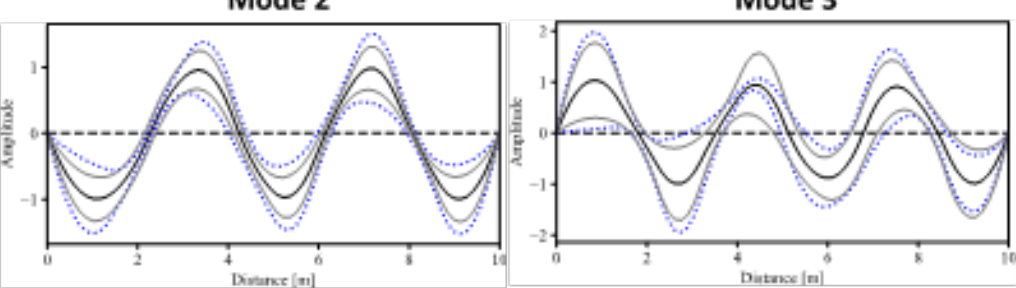

Mode 4

Mode 5

Mode 6

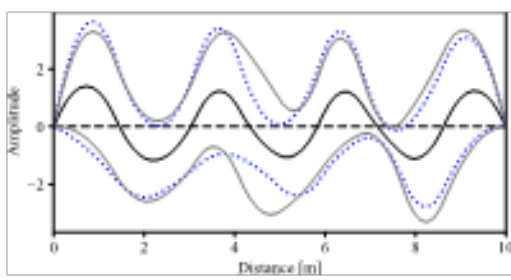

Mode 7

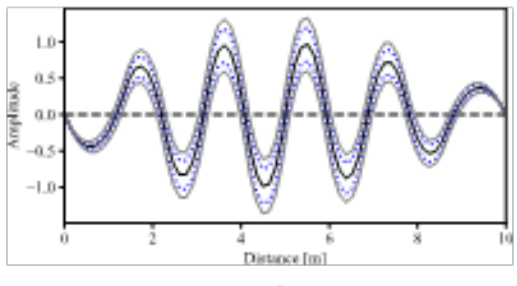

Mode 10

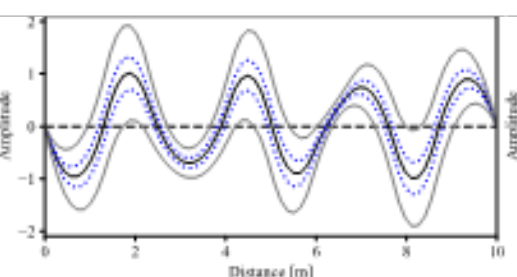

Mode 8

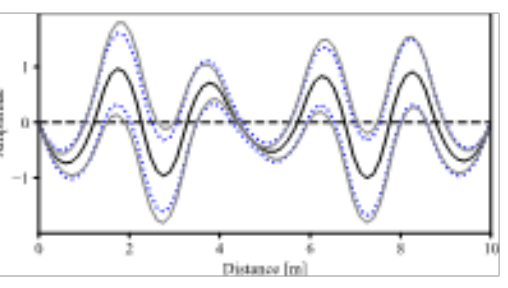

Mode 9

Figure 5. Confidence intervals of mode shapes. 


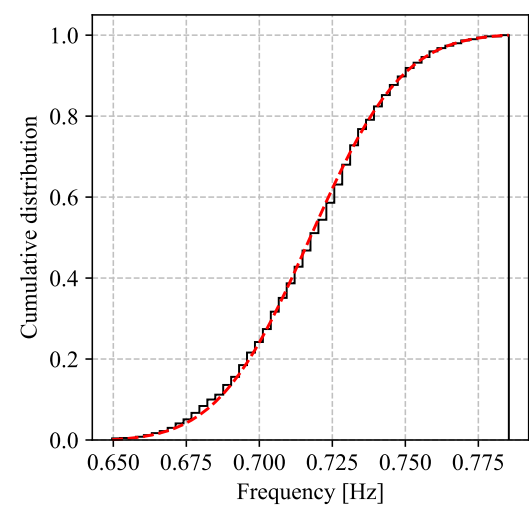

Mode 1

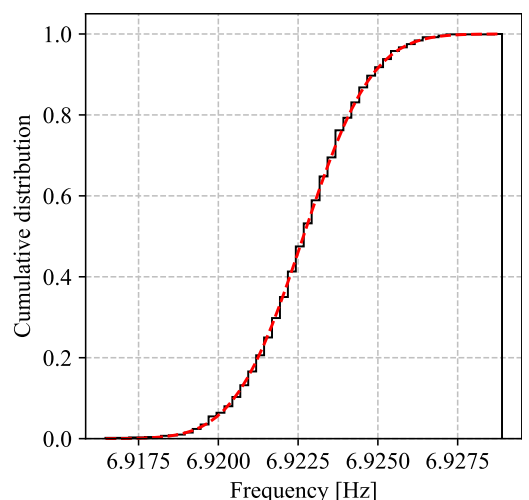

Mode 4

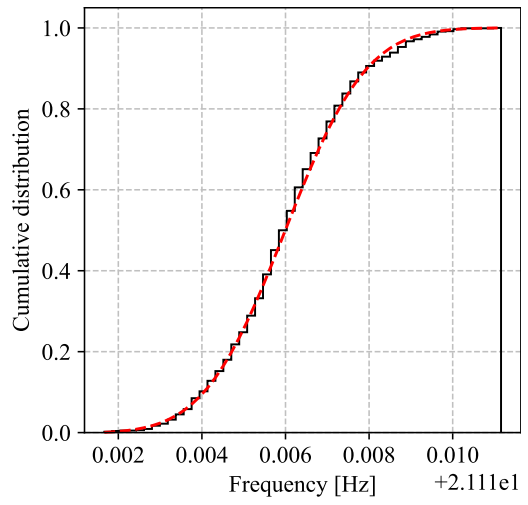

Mode 7

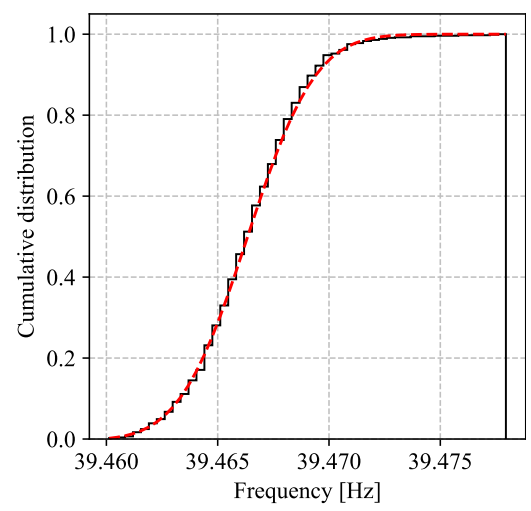

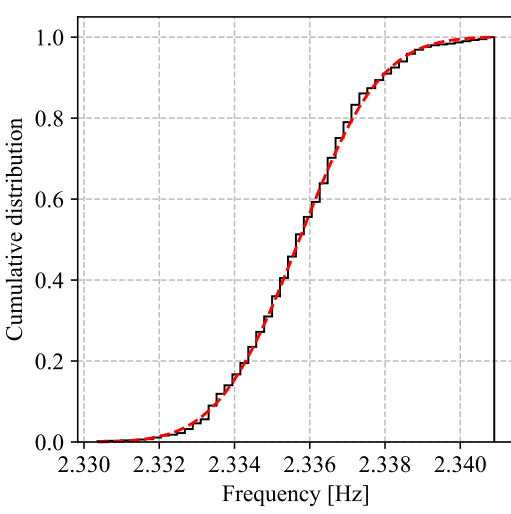

Mode 2

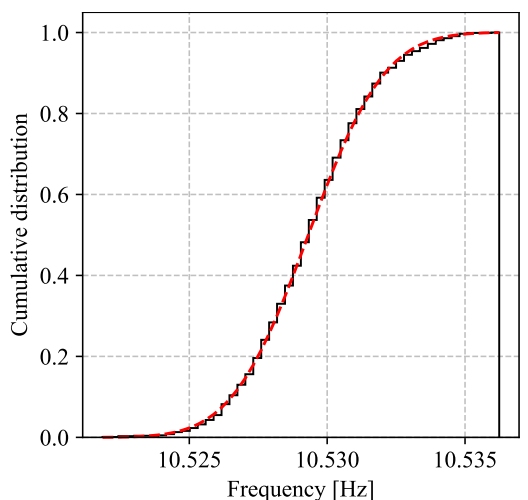

Mode 5

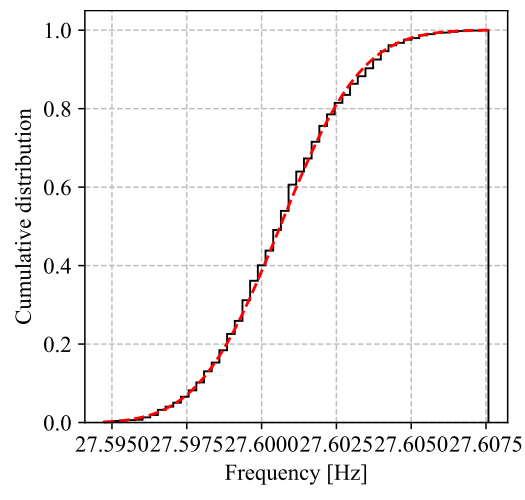

Mode 8

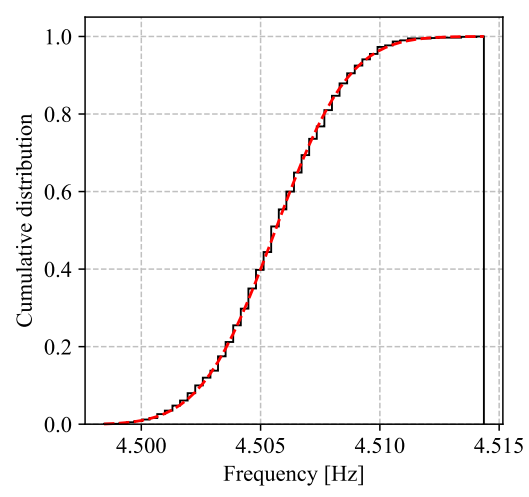

Mode 3

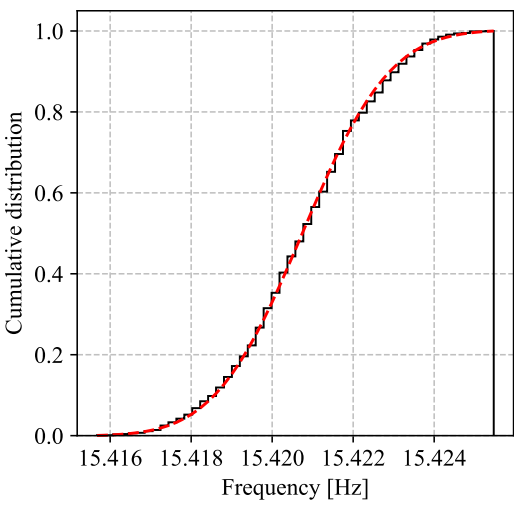

Mode 6

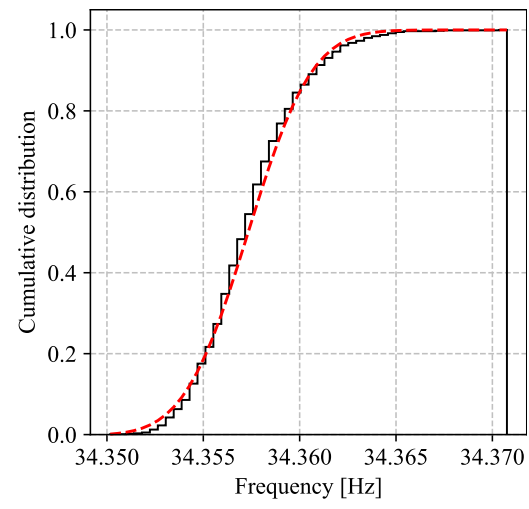

Mode 9

Mode 10

Figure 6. Example 1: Empirical cumulative distribution function of natural frequencies and normal cumulative distribution constructed from the sample mean and sample variance. 


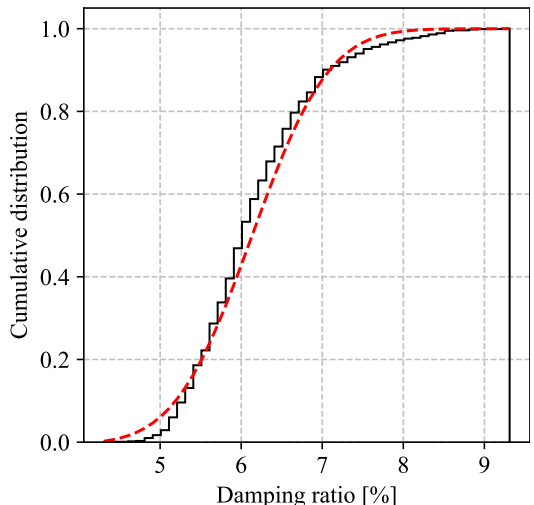

Mode 1

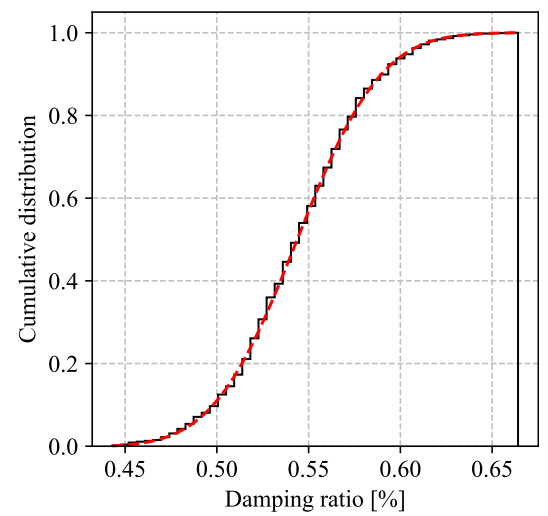

Mode 4

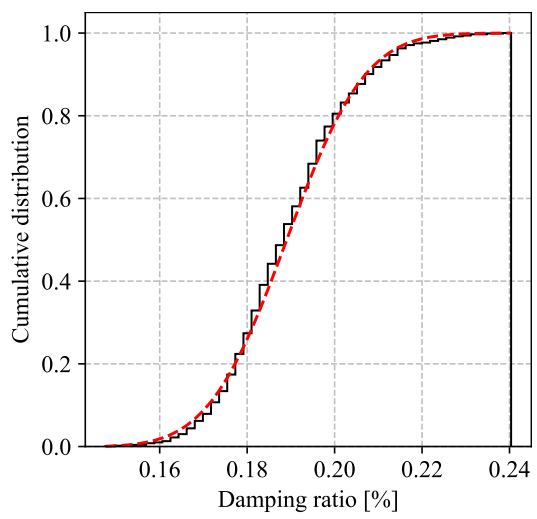

Mode 7

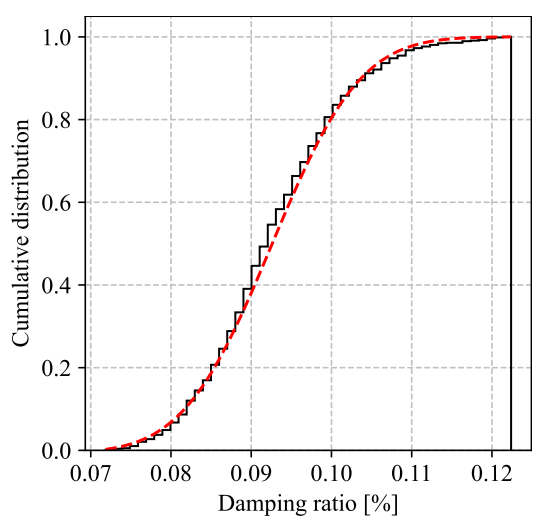

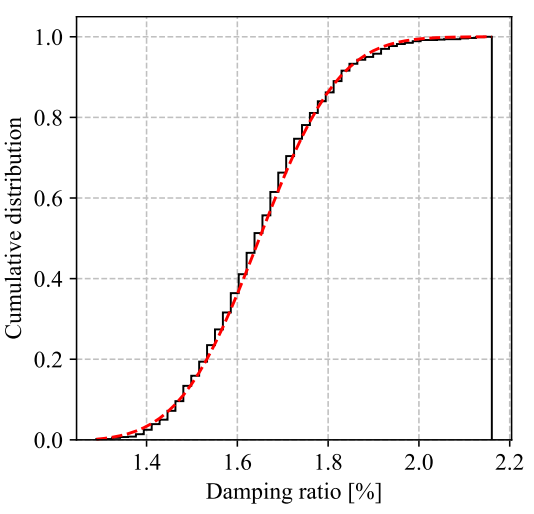

Mode 2

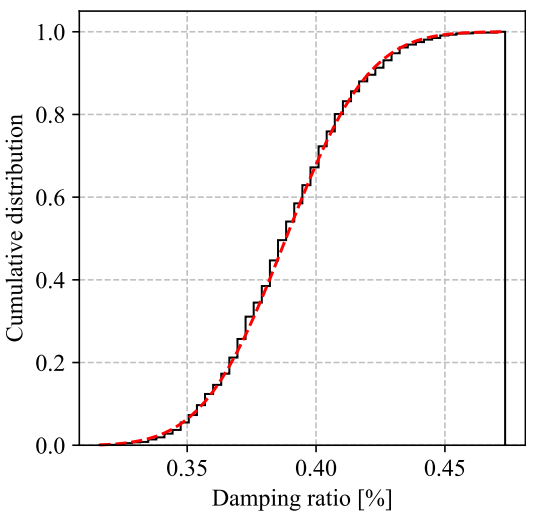

Mode 5

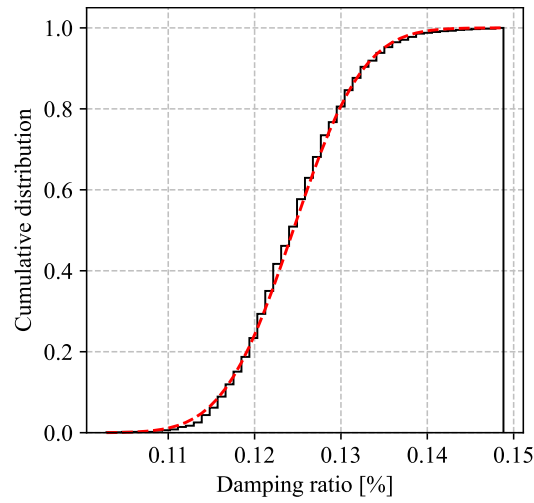

Mode 8

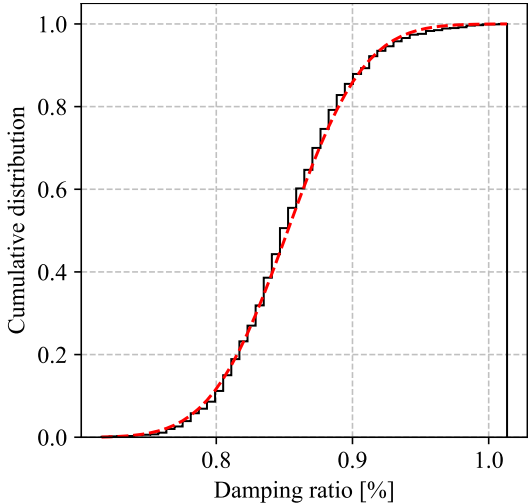

Mode 3

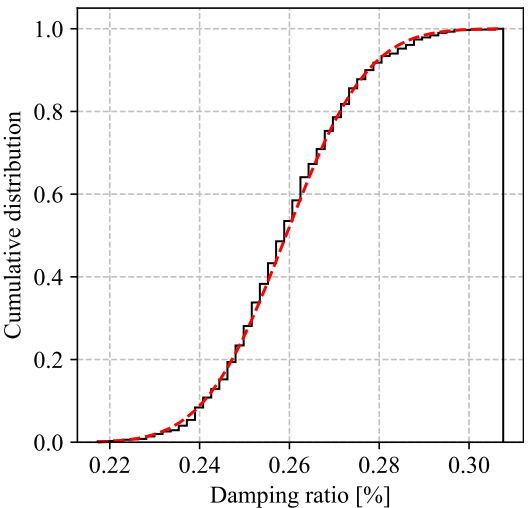

Mode 6

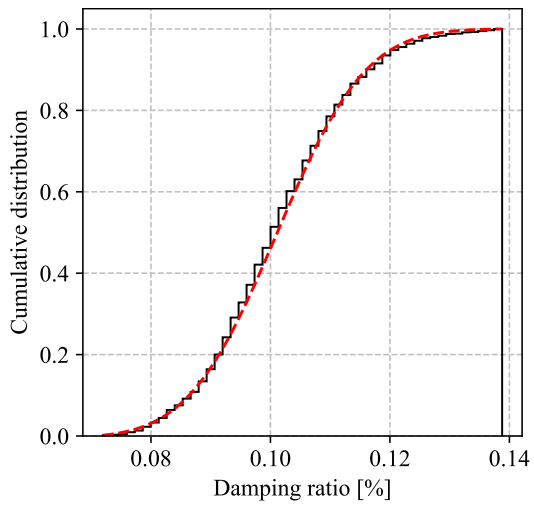

Mode 9

Mode 10

Figure 7. Example 1: Empirical cumulative distribution function of damping ratios and normal cumulative distribution constructed from the sample mean and sample variance. 


\subsection{Validation with data from ambient vibration test}

This section validates the confidence intervals of the modal parameters of the F-17/18 concrete block (see Figure 8) of the Itaipú Hydroelectric Dam. This concrete block is located on stretch F of the main dam. It is equipped with two triaxial accelerometers ACL05-F18 (station 5GS6) and ACL06-F18 (station 5GS8), which are configured to acquire signals at a frequency of $200 \mathrm{~Hz}$ and produce hourly .gcf files (Guralp compressed file). For this example, records were collected from 01/07/2018 from 02:00:00 to 02:59:599, for a total of 720000 samples in each direction (longitudinal, transverse and vertical), as can be seen in Figure 9. These time series were decimated by a factor of 5 to obtain a final sample frequency of $40 \mathrm{~Hz}$, thus reducing the total number of samples to 144000 .

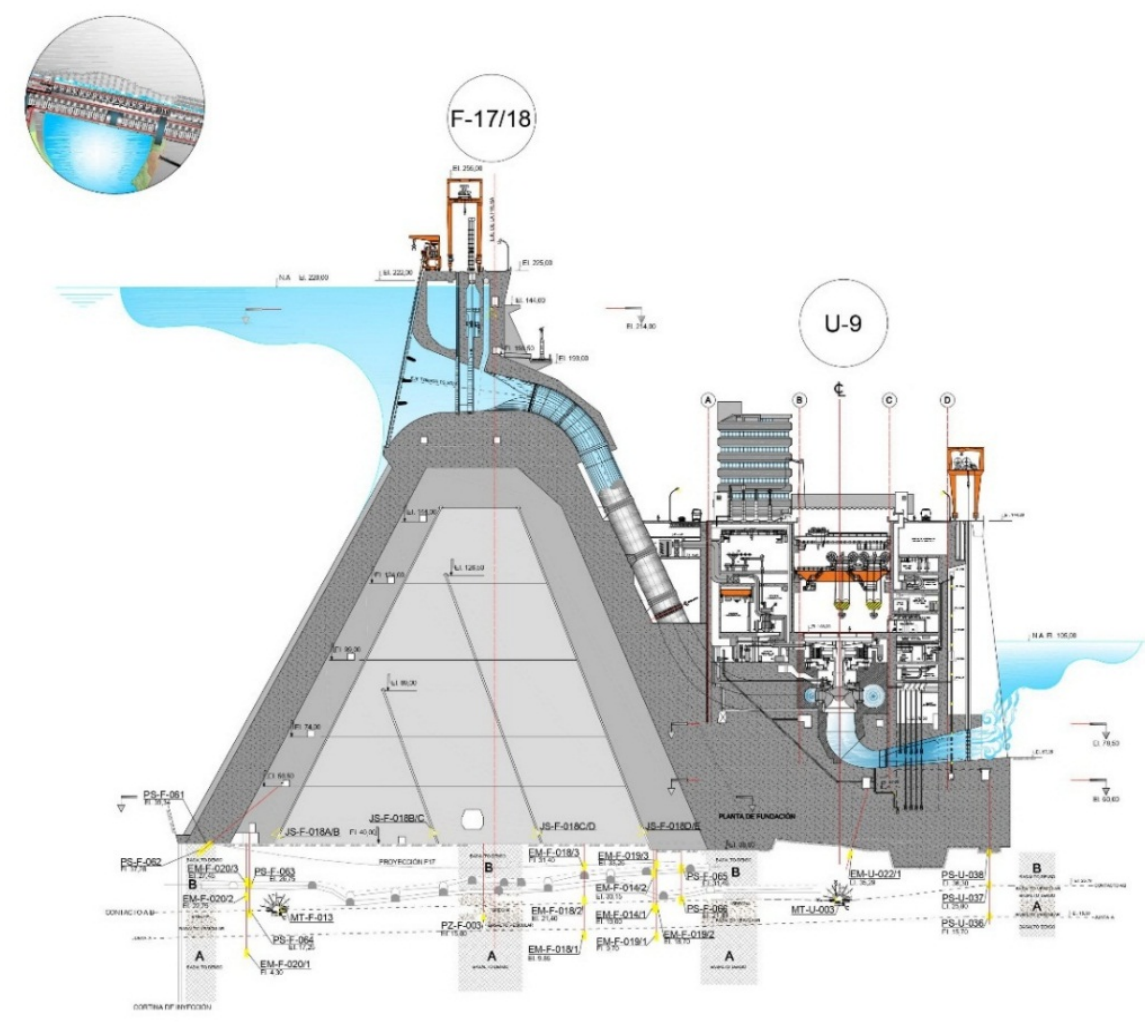

Figure 8. Cross-section of the F-17/18 concrete block
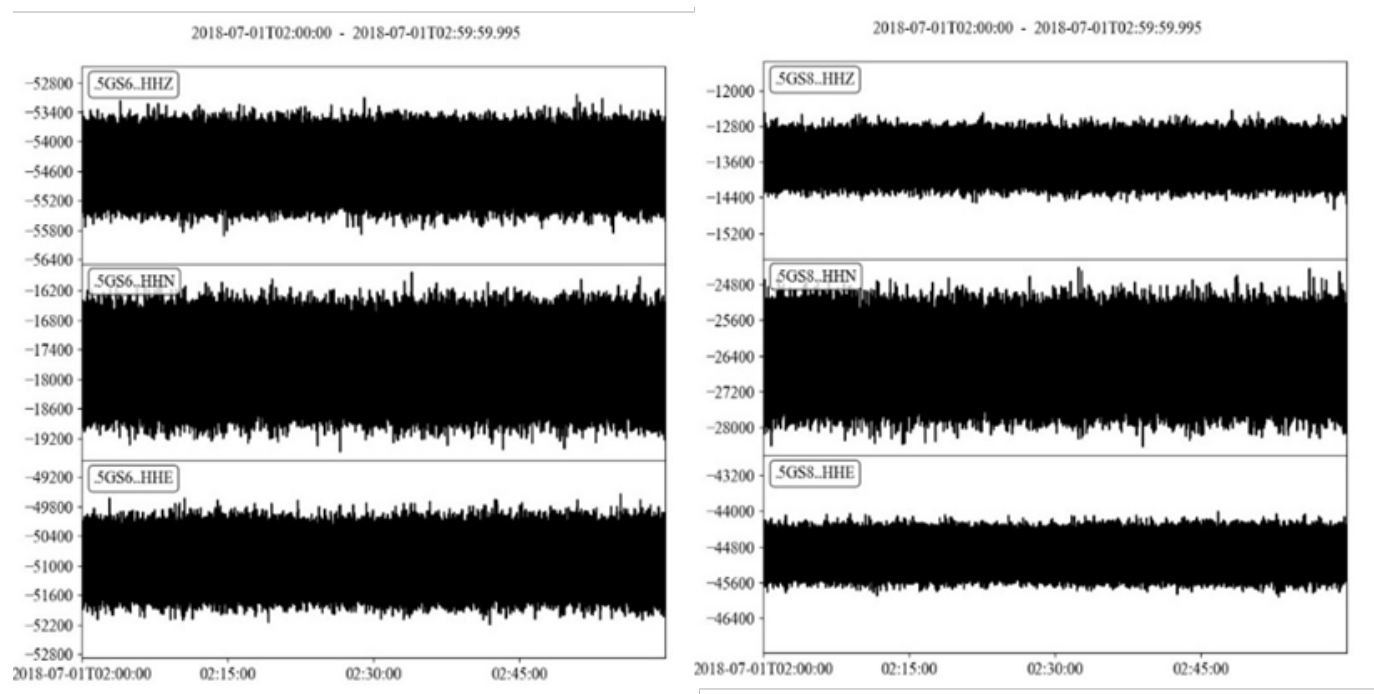

Figure 9. Measured responses caused by environmental vibrations. Left (ACL05-F18), (Right ACL06-F18). 
The SSI-COV method uses the following parameters: the number of blocks of the Toeplitz matrix $T_{1 i}$ was set to $i=80$, and for the construction of the stability diagram, a range of orders from $n=2$ to $n=160$ in increments of 2 was used. To quantify the uncertainties with approach 1 , a number of blocks $n_{b}=200$ was used. Each block has a length of $N_{b}=720$ samples, which amounts to $18 \mathrm{~s}$.

The resulting stability diagrams are more polluted by poles because the noise is not controlled in this application. Figure 10 (a) shows the stability diagram constructed immediately after the cleaning stage. It can be seen that with the adopted methodology for cleaning the stability diagram, the number of poles was significantly reduced. Some of the probably physical poles remaining after this stage have large standard deviations (grey horizontal bars). However, Figure 10 (b) shows that the resulting physical poles have minor standard deviations. Therefore, in this example, the standard deviations from approach 1 agree well with the expected results. The modal parameters information for the physical modes, represented by the red dots in the stability diagram in Figure 10 (b), is presented in Table 4. All vibration modes show an MPC close to 1 , indicating a high degree of physicality.
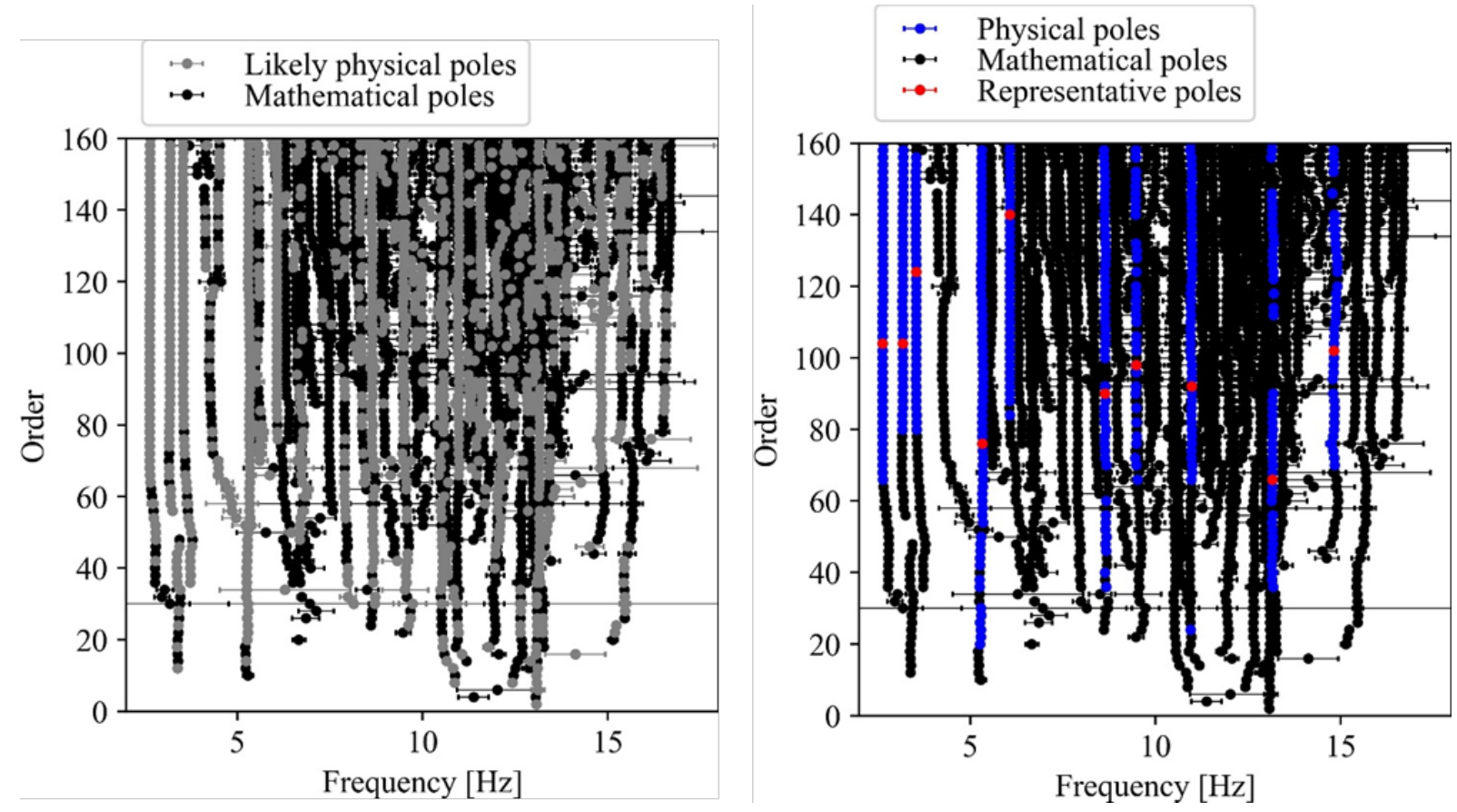

Figure 10. Example 2: (a) Stability diagram after the cleaning stage. (b) Stability diagram after the MPE procedure

Table 4. Modal parameters identified in the F-17/18 concrete block

\begin{tabular}{ccccccccccc}
\hline & Mode 1 & Mode 2 & Mode 3 & Mode 4 & Mode 5 & Mode 6 & Mode 7 & Mode 8 & Mode 9 & Mode 10 \\
\hline $\begin{array}{c}\text { Frequency } \\
\text { [Hz] }\end{array}$ & 2.639 & 3.172 & 3.530 & 5.302 & 6.064 & 8.611 & 9.471 & 10.956 & 13.075 & 14.831 \\
$\begin{array}{c}\text { Damping } \\
\text { ratio [\%] }\end{array}$ & 3.917 & 2.634 & 3.684 & 3.451 & 0.306 & 1.395 & 0.492 & 0.554 & 1.732 & 1.329 \\
MPC & 0.937 & 0.981 & 0.998 & 0.977 & 0.973 & 0.953 & 0.943 & 0.932 & 0.899 & 0.947 \\
\hline
\end{tabular}

To apply the Bootstrap technique, 1000 pseudo time series were generated using a length $l=24000$. Figures 11 and 12 compare the empirical cumulative distribution of the samples obtained for the natural frequencies and damping ratios, respectively, with the normal distribution constructed from each sample's means and standard deviations. It can be observed that most vibration modes fit to a normal distribution. Tables 5 and 6 present the confidence intervals obtained by the two approaches for the natural frequencies and the damping ratios, respectively. At first glance, it can be seen that the standard deviations obtained for the damping ratios are larger than those obtained for the natural frequencies. This is because the damping ratios are a parameter that is highly sensitive to the quality of the covariance matrices and the SSI-COV parameters, such as the number of blocks rows 
of the Toeplitz matrix. Although not precisely equal, the confidence intervals obtained by the two approaches are consistent in their magnitude.

Additionally, it is essential to report that the propagation method demonstrated a computational processing time drastically inferior to the Boostrap technique in both examples applied in this work. The procedures used in this article were implemented in the Python programming language and executed on Intel(R) Core(TM) i7-8700k CPU $3.70 \mathrm{GHz}$ with $32 \mathrm{GBytes}$. The computational time used for the concrete block example was 30 seconds to obtain the standard deviations of all modes in the multi-order model (approach 1) and 5 seconds to get the modal parameters of each bootstrap time series, adding up to a total time of approximately 83 min for Bootstrap technique.

Table 5. Example 2: Confidence intervals of natural frequencies

\begin{tabular}{|c|c|c|c|c|c|c|c|c|}
\hline \multirow{3}{*}{ Mode } & \multicolumn{5}{|c|}{ Bootstrap verification } & \multicolumn{3}{|c|}{$\begin{array}{l}\text { Uncertainty quantification from } \\
\text { (Reynders et al., 2008; Döhler et al., } \\
\text { 2013) }\end{array}$} \\
\hline & \multirow[t]{2}{*}{ Estimate } & \multirow[t]{2}{*}{ Bias } & \multirow{2}{*}{$\begin{array}{l}\text { Standard } \\
\text { Deviation }\end{array}$} & \multicolumn{2}{|c|}{$\begin{array}{c}\text { 95\% Confidence } \\
\text { intervals }\end{array}$} & \multirow{2}{*}{$\begin{array}{l}\text { Standard } \\
\text { Deviation }\end{array}$} & \multicolumn{2}{|c|}{$\begin{array}{c}\text { 95\% Confidence } \\
\text { intervals }\end{array}$} \\
\hline & & & & Lower & Upper & & Lower & Upper \\
\hline 1 & 2.6369 & 2.10E-03 & 0.0062 & 2.6245 & 2.6493 & 0.0081 & 2.6228 & 2.6552 \\
\hline 2 & 3.1740 & $2.10 \mathrm{E}-03$ & 0.0070 & 3.1600 & 3.1880 & 0.0064 & 3.1591 & 3.1847 \\
\hline 3 & 3.5288 & $9.60 \mathrm{E}-04$ & 0.0067 & 3.5154 & 3.5422 & 0.0123 & 3.5052 & 3.5544 \\
\hline 4 & 5.2920 & $9.81 \mathrm{E}-03$ & 0.0209 & 5.2502 & 5.3338 & 0.0270 & 5.2478 & 5.3558 \\
\hline 5 & 6.067 & $2.90 \mathrm{E}-03$ & 0.0061 & 6.0551 & 6.0795 & 0.0114 & 6.0415 & 6.0873 \\
\hline 6 & 8.6420 & $3.14 \mathrm{E}-02$ & 0.0196 & 8.6028 & 8.6812 & 0.0204 & 8.5699 & 8.6513 \\
\hline 7 & 9.4920 & $2.15 \mathrm{E}-02$ & 0.0215 & 9.4490 & 9.5350 & 0.0355 & 9.3996 & 9.5414 \\
\hline 8 & 10.9530 & 3.30E-03 & 0.0130 & 10.9270 & 10.9790 & 0.0154 & 10.9254 & 10.9872 \\
\hline 9 & 13.1070 & $3.21 \mathrm{E}-02$ & 0.0562 & 12.9946 & 13.2194 & 0.0100 & 13.0550 & 13.0948 \\
\hline 10 & 14.8640 & $3.34 \mathrm{E}-02$ & 0.1750 & 14.5140 & 15.2140 & 0.1387 & 14.5532 & 15.1080 \\
\hline
\end{tabular}

Table 6. Example 2: Confidence intervals of damping ratios

\section{Bootstrap verification}

\begin{tabular}{|c|c|c|c|c|c|c|c|c|}
\hline \multirow{3}{*}{ Mode } & \multirow{3}{*}{ Estimate } & \multirow{3}{*}{ Bias } & \multirow{3}{*}{$\begin{array}{l}\text { Standard } \\
\text { Deviation }\end{array}$} & \multirow{2}{*}{\multicolumn{2}{|c|}{$\begin{array}{l}\text { 95\% Confidence } \\
\text { intervals }\end{array}$}} & \multirow{3}{*}{$\begin{array}{l}\text { Standard } \\
\text { Deviation }\end{array}$} & \multicolumn{2}{|l|}{ 2013) } \\
\hline & & & & & & & \multicolumn{2}{|c|}{$\begin{array}{l}\text { 95\% Confidence } \\
\text { intervals }\end{array}$} \\
\hline & & & & Lower & Upper & & Lower & Upper \\
\hline 1 & 4.5410 & $6.24 \mathrm{E}-01$ & 0.4350 & 3.6710 & 5.4110 & 0.5447 & 2.8277 & 5.0063 \\
\hline 2 & 2.9190 & 2.85E-01 & 0.4410 & 2.0370 & 3.8010 & 0.3210 & 1.9919 & 3.2761 \\
\hline 3 & 3.4120 & $2.72 \mathrm{E}-01$ & 0.3210 & 2.7700 & 4.0540 & 0.4214 & 2.8409 & 4.5265 \\
\hline 4 & 3.6820 & 2.31E-01 & 0.4460 & 2.7900 & 4.5740 & 0.7103 & 2.0303 & 4.8717 \\
\hline 5 & 0.3215 & $1.55 \mathrm{E}-02$ & 0.1370 & 0.0475 & 0.5955 & 0.1496 & 0.0067 & 0.6053 \\
\hline 6 & 1.3743 & $2.02 \mathrm{E}-02$ & 0.3418 & 0.6907 & 2.0579 & 0.2864 & 0.8217 & 1.9674 \\
\hline 7 & 0.4095 & 8.29E-02 & 0.1525 & 0.1045 & 0.7145 & 0.1359 & 0.2206 & 0.7641 \\
\hline 8 & 0.5869 & 3.29E-02 & 0.1312 & 0.3245 & 0.8493 & 0.1452 & 0.2636 & 0.8444 \\
\hline 9 & 1.2578 & 4.74E-01 & 0.3699 & 0.5180 & 1.9976 & 0.2147 & 1.3026 & 2.1615 \\
\hline 10 & 1.3456 & $1.59 \mathrm{E}-02$ & 0.3010 & 0.7436 & 1.9476 & 0.5473 & 0.2351 & 2.4242 \\
\hline
\end{tabular}
(Reynders et al., 2008; Döhler et al., 2013)

Uncertainty quantification from 


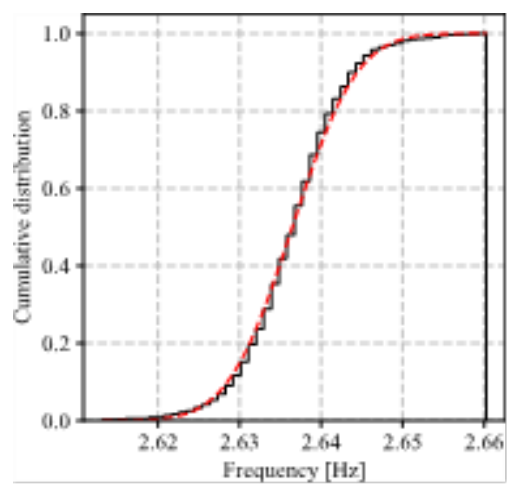

Mode 1

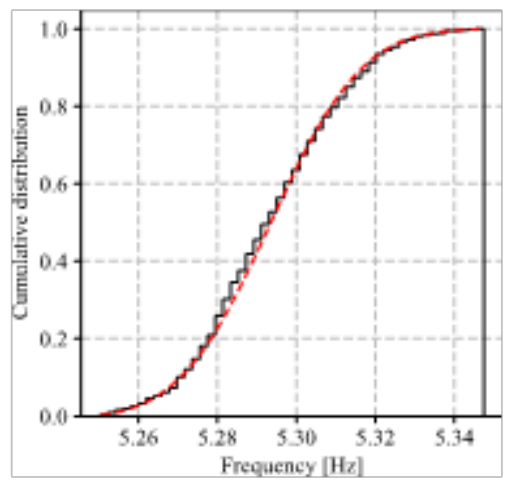

Mode 4

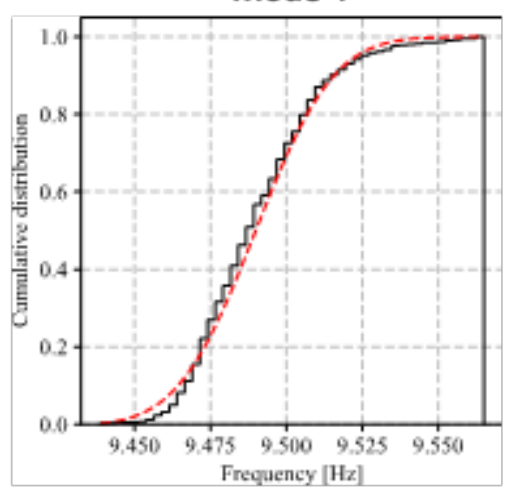

Mode 7

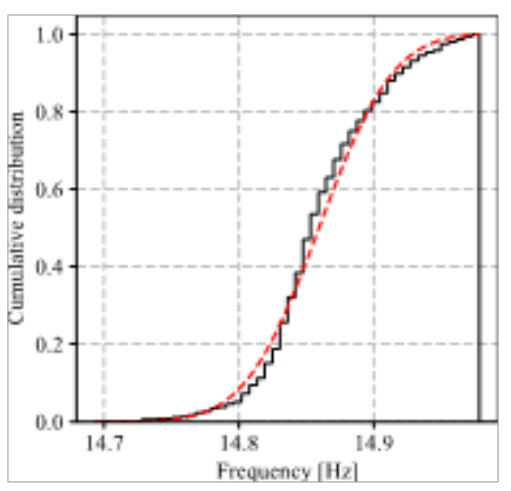

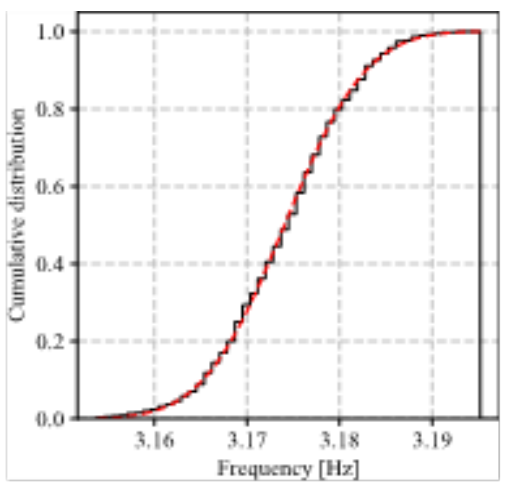

Mode 2

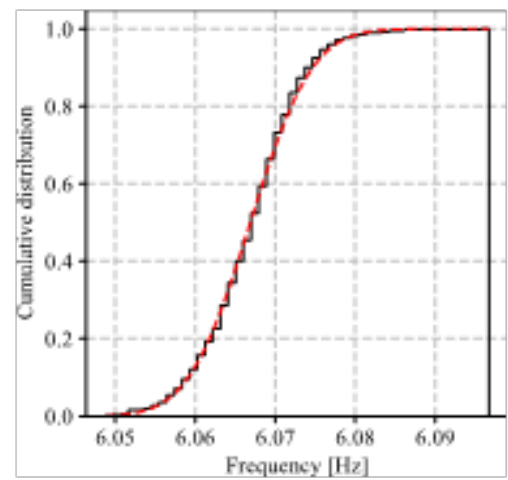

Mode 5

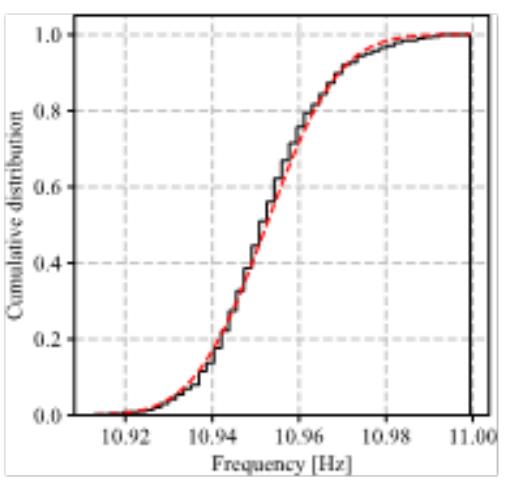

Mode 8

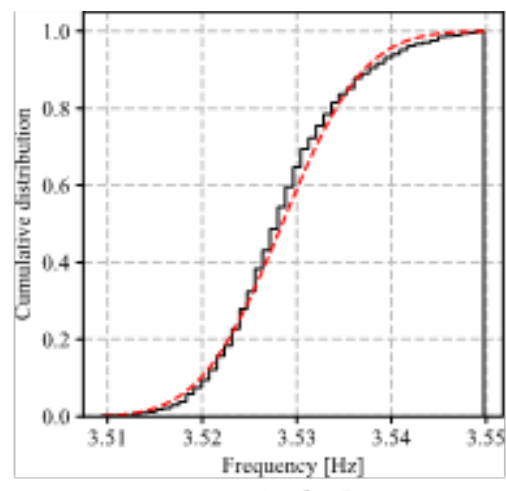

Mode 3

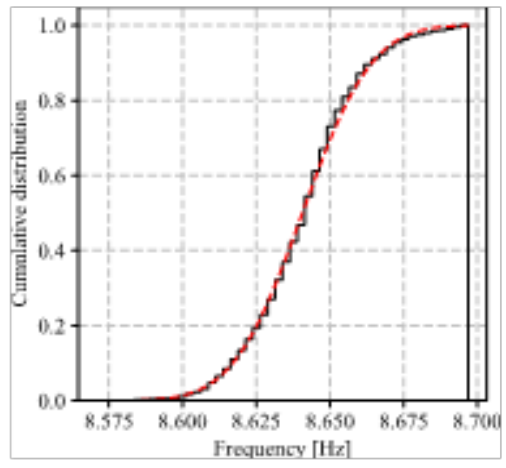

Mode 6

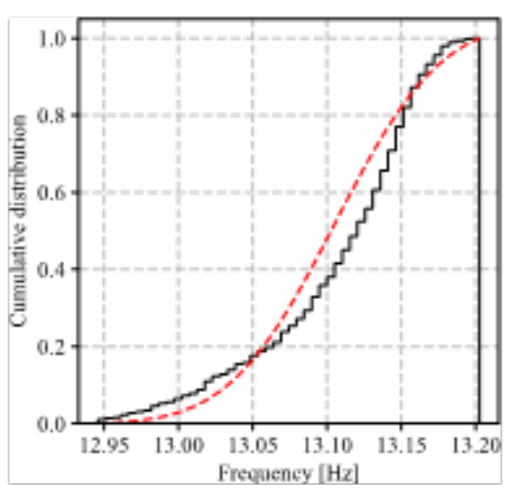

Mode 9

Mode 10

Figure 11. Example 2: Empirical cumulative distribution function of natural frequencies and normal cumulative distribution constructed from the sample mean and sample variance. 


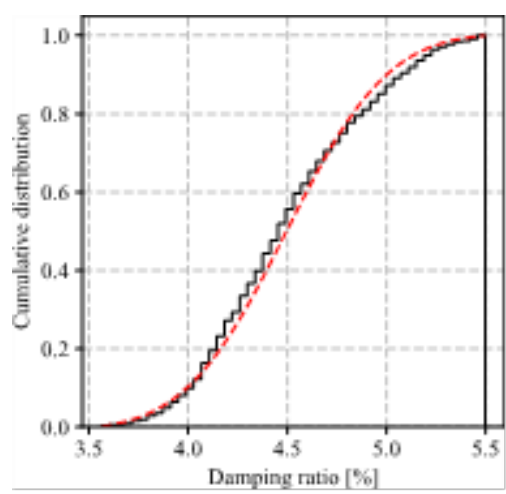

Mode 1

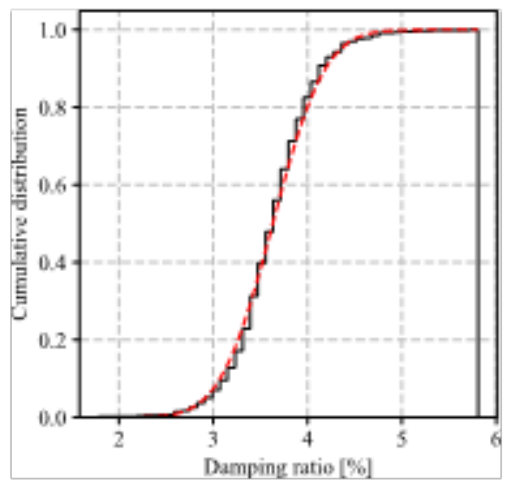

Mode 4

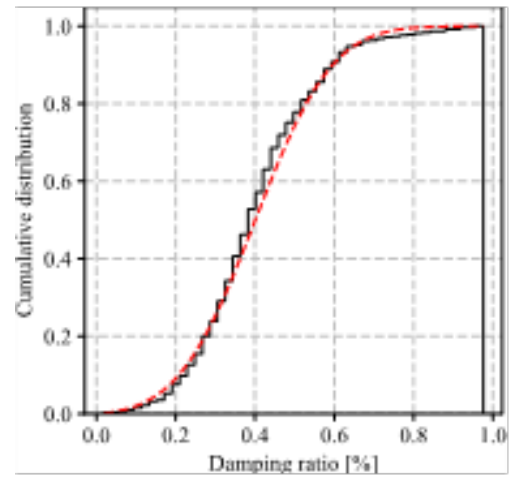

Mode 7

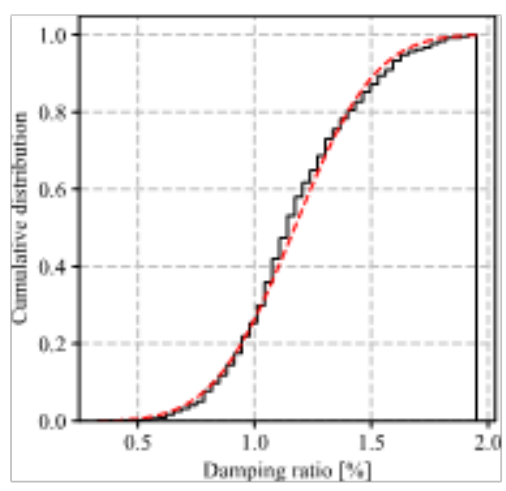

Mode 10

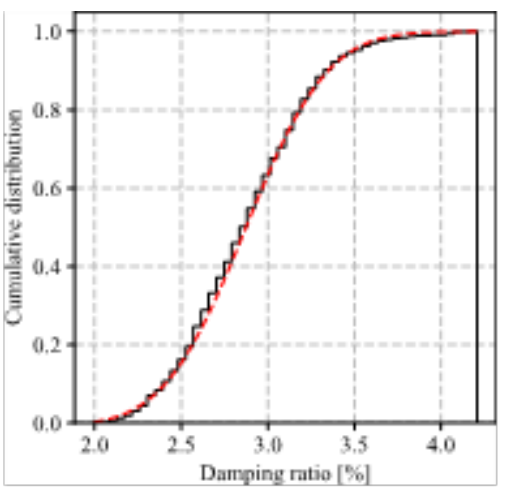

Mode 2

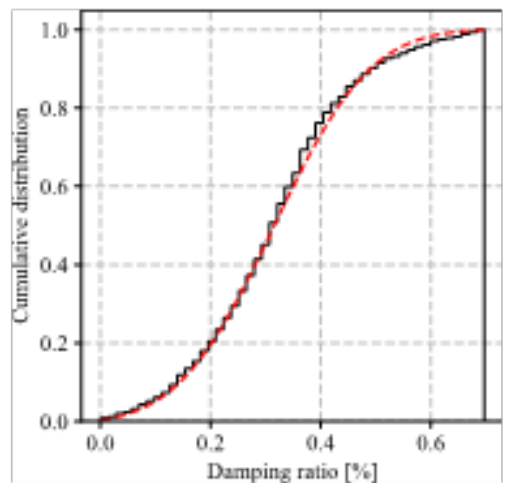

Mode 5

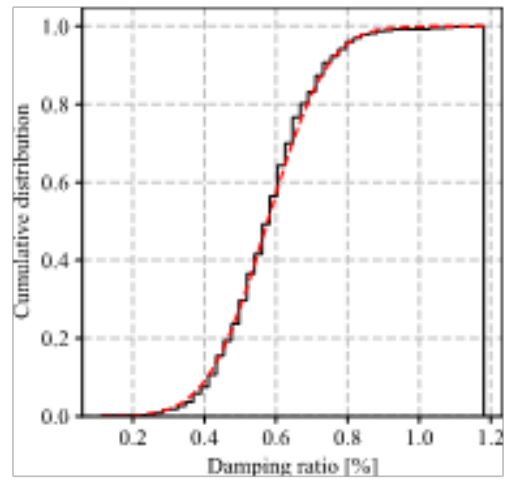

Mode 8

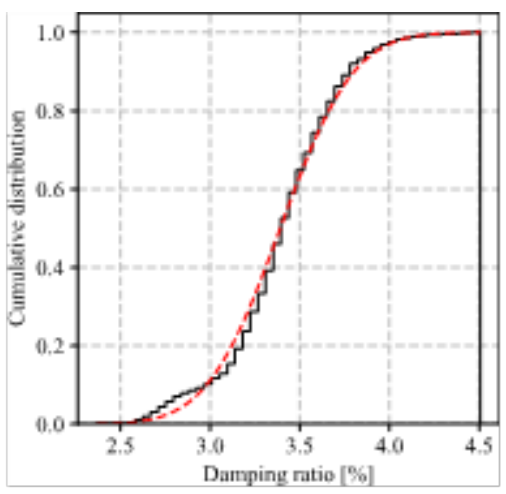

Mode 3

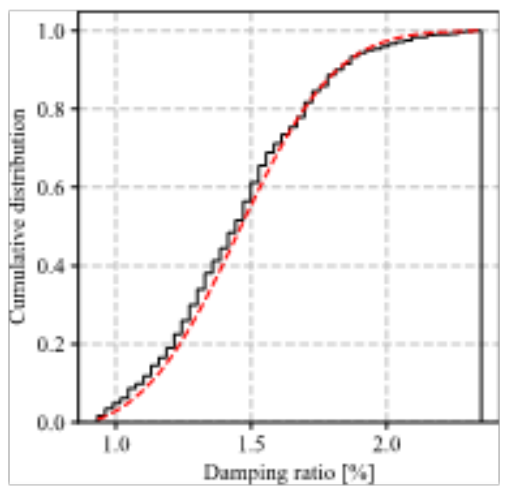

Mode 6

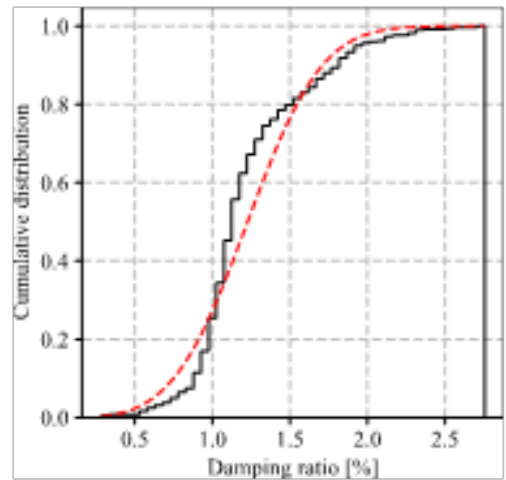

Mode 9

Figure 12. Example 2: Empirical cumulative distribution function of damping ratios and normal cumulative distribution constructed from the sample mean and sample variance 


\section{CONCLUSIONS}

In this work, the Bootstrap technique was used to validate the confidence intervals obtained from the approach proposed by (Reynders et al., 2008; Döhler et al., 2013), which is based on the propagation of the uncertainties of the collected data through all stages of the SSI-COV method. In the bootstrap technique, the response due to ambient vibrations is resampled to generate a bootstrap set of pseudo time series used to perform the stochastic subspaces and obtain a bootstrap set of modal parameters. Therefore, the computational time used by the bootstrap method to quantify the uncertainty is high. However, being a numerical method and easy to apply, it is a powerful tool in evaluating uncertainty. Furthermore, the experiments with simulated and real data showed similarities in evaluating uncertainties in the modal parameters by the two methodologies. Therefore, it is possible to conclude that the approach proposed by (Reynders et al., 2008; Döhler et al., 2013), provides reliable estimates of the covariances for the modal parameters with a very low computational cost compared to the Bootstrap technique. Futhermore, applying this procedure to estimate uncertainties in the SSI-COV method becomes attractive for the continuous dynamic monitoring of structures.

\section{Acknowledgments}

The authors would like to thank UNILA ("Universidade da Integração Latino Americana"), FPTI ("Fundação Parque Tecnológico Itaipu") for the financial support and and EB.DT ("Centro de Competência em Estruturas de Barragens") for laboratories to perform the simulations and Itaipu Binacional for the availability of the data.

Author Contributions: Conceptualization, Y. Ardila, I. Gómez and J. Villalba; Methodology, Y. Ardila, I. Gómez and J. Villalba; Investigation, Y. Ardila, I. Gómez and J. Villalba; Writing-review \& editing, Y. Ardila, I. Gómez J. Villalba, and L. Aracayo; Supervision, I. Gómez and J. Villalba, Funding adquisition I. Gomez, and L. Aracayo.

Editor: Marcílio Alves.

\section{References}

Magalhães, F., Caetano, E., \& Cunha, Á. (2008). Operational modal analysis and finite element model correlation of the Braga Stadium suspended roof. Engineering Structures, 30(6), 1688-1698.

Brownjohn, J. M. W., Magalhaes, F., Caetano, E., \& Cunha, A. (2010). Ambient vibration re-testing and operational modal analysis of the Humber Bridge. Engineering Structures, 32(8), 2003-2018.

Liu, Y.-C., Loh, C.-H., \& Ni, Y.-Q. (2012). Stochastic subspace identification for output-only modal analysis: application to super high-rise tower under abnormal loading condition. Earthquake Engineering \& Structural Dynamics, 42(4), 477-498.

Pereira, S., Magalhães, F., Gomes, J. P., Cunha, Á., \& Lemos, J. V. (2018). Dynamic monitoring of a concrete arch dam during the first filling of the reservoir. Engineering Structures, 174, 548-560.

Reynders, E., Pintelon, R., \& De Roeck, G. (2008). Uncertainty bounds on modal parameters obtained from stochastic subspace identification. Mechanical Systems and Signal Processing, 22(4), 948-969.

Pereira, S., Reynders, E., Magalhães, F., Cunha, Á., \& Gomes, J. P. (2020). The role of modal parameters uncertainty estimation in automated modal identification, modal tracking, and data normalization. Engineering Structures, 224, 111208.

Döhler, M., Marin, L., Mevel, L., \& Bernal, D. (2015). Operational modal analysis with uncertainty quantification for SDDLVbased damage localization. MATEC Web of Conferences, 20, 02002.

Verboven, P., Cauberghe, B., Parloo, E., Vanlanduit, S., \& Guillaume, P. (2004). User-assisting tools for a fast frequency-domain modal parameter estimation method. Mechanical Systems and Signal Processing, 18(4), 759-780.

Wu, G., He, M., Liang, P., Ye, C., \& Xu, Y. (2020). Automated Modal Identification Based on Improved Clustering Method. Mathematical Problems in Engineering, 2020.

Döhler, M., \& Mevel, L. (2013). Efficient multi-order uncertainty computation for stochastic subspace identification. Mechanical Systems and Signal Processing, 38(2), 346-366. 
E. Reynders, J. Houbrechts, and G. De Roeck. (2012). "Fully automated (operational) modal analysis". Mechanical Systems and Signal Processing, vol. 29, 228-250.

Antsaklis, P.J.; Astolfi, A. (2014). Realizations in Linear Systems Theory. In: Baillieul J., Samad T. (eds) Encyclopedia of Systems and Control. Springer, London.

Akaike, H. (1974). Stochastic theory of minimal realization. IEEE Transactions on Automatic Control, 19, 667-674.

Aoki, M. (1987). State-Space Modelling of Time Series. Springer-Verlag, Berlin, Germany.

Arun, K. S.; Kung, S. Y. (1990). Balanced approximation of stochastic systems. SIAM Journal on Matrix Analysis and Applications, 11. pp. 42-68.

Oppenheim, A.V.; Schafer, R. W. (1975). Digital Signal Processing, Prentice-Hall.

Kung S.Y. (1978). A new identification and model reduction algorithm via singular value decomposition. In Proceedings of the 12th Asilomar Conference on Circuits, Systems and Computers, pp. 705-714, Asilomar, CA, USA.

Zeiger, H.P.; Mcewen A.J. (1974). Approximate linear realization of given dimension via Ho's algorithm. IEEE Transactions on Automatic Control, AC-19(2), 153.

Efron, B., (1979). Bootstrap methods: Another look at the jackknife. Annals of Statistics, vol. 7, pp. 1-26.

Kunsch, H. R., (1979). The jackknife and the bootstrap for general stationary observations. Annals of Statistics, vol. 17(3), pp. 1217-1241.

Giampellegrini, L. (2007). Uncertainty in Correlation-Driven Operational Modal Estimation. Doctoral Thesis. University of London. 